\title{
International-vergleichende Forschung zur Bildungspolitik: Literatur-Review theoretischer Ansätze und Perspektiven der vergleichenden Politikwissenschaft
}

\author{
Carolin Knauber iD
}

Eingegangen: 13. Juni 2017 / Angenommen: 4. August 2017 / Online publiziert: 24. August 2017 (C) Der/die Autor(en) 2017. Dieser Artikel ist eine Open-Access-Publikation.

Zusammenfassung In der international-vergleichenden Bildungs- und Erwachsenenbildungsforschung finden Fragen der Bildungspolitik und insbesondere der Politik zur Erwachsenenbildung derzeit mehr und mehr Beachtung. In der vergleichenden Politikwissenschaft ist Bildungspolitik ein bereits gut etabliertes, wenngleich ebenfalls noch junges Forschungsfeld. Daher zielt dieser Literatur-Review darauf, in der vergleichenden Politikwissenschaft verwendete Ansätze und Theorien auf ihren Anregungsgehalt für die international-vergleichende Erwachsenenbildungsforschung zu prüfen. Der Review bietet eine systematische Analyse von Veröffentlichungen, die sich mit bildungspolitischen Themen international-vergleichend beschäftigen und dabei Ansätze und Theorien der vergleichenden Politikwissenschaft verwenden. Dem Beitrag liegt eine systematische Literaturrecherche in einschlägigen Datenbanken für den Zeitraum von 2006 bis 2016 zugrunde. Insgesamt flossen 65 Titel ein. In diesen Veröffentlichungen wurden die folgenden Theorien und Ansätze identifiziert: Wohlfahrtsstaatentheorie, Varieties of Capitalism, Parteiendifferenztheorie, Globalisierung und Europäisierung, Pfadabhängigkeit sowie Akteurzentrierter Institutionalismus. Der vorliegende Review skizziert sowohl die verwendeten theoretischen Zugänge und Ansätze als auch die darauf basierenden Forschungsergebnisse. Darauf aufbauend werden Anknüpfungsmöglichkeiten, Erträge und auch Einschränkungen dieser Theorien und Ansätze für die international-vergleichende Erwachsenenbildungsforschung sowie das Themenfeld der Erwachsenenbildungspolitik diskutiert.

Schlüsselwörter Literaturreview · Internationaler Vergleich · Bildungspolitik · Theorien · Politikwissenschaft · Interdisziplinär

C. Knauber $(\bowtie)$

Abteilung „System und Politik“, Deutsches Institut für Erwachsenenbildung - Leibniz-Zentrum für Lebenslanges Lernen e. V., Heinemannstr. 12-14, 53175 Bonn, Deutschland

E-Mail: knauber@die-bonn.de 


\title{
International-Comparative Research on Education Policy: A Review Article on Theoretical Approaches from Comparative Politics
}

\begin{abstract}
The field of education policy, in particular adult education policy, is gaining importance in recent international-comparative research on education and adult education. In comparative politics, education policy is also a young but increasingly established issue. The close connection of education policy to the discipline of comparative politics, as well as theories used in that discipline, provide a wellfounded research approach on education policy, which should be considered by international-comparative research on adult education. This paper is a literature review, systematically analysing publications dealing with educational policy topics in an international-comparative perspective, using approaches and theories from the discipline of comparative politics. The contribution is based on a systematic literature research in relevant databases for the period 2006 to 2016. 65 titles were included in this literature review. The theories and approaches identified in the publications include the Welfare State Theory, Varieties of Capitalism, Partisan Theory, Globalisation and Europeanisation, Path dependency, and Actor-centered Institutionalism. This paper presents the theoretical approaches used in the publications and outlines their research results. Additionally, the paper discusses limitations, potential use and benefits of these theories and approaches for international-comparative research on adult education policy.
\end{abstract}

Keywords Review · International comparison - Educational policy · Theories · Political sciences $\cdot$ Interdisciplinary

\section{Relevanz für die Erwachsenenbildungsforschung}

Gegenwärtig gewinnt die international-vergleichende Forschung zur Bildungspolitik an Bedeutung und Produktivität. Mit der fortschreitenden Globalisierung und der zugleich abnehmenden Bedeutung des Nationalstaats sowie der steigenden Relevanz inter- und supranationaler Organisationen (EU, OECD, UNESCO), die sich (auch) mit Bildung beschäftigen, sind die Möglichkeiten für und das Interesse an international-vergleichende(r) Forschung gewachsen. Vergleicht man die ältere mit der jüngeren international-vergleichenden Bildungspolitikforschung, so wird eine zunehmende theoretische Fundierung der Forschung erkennbar.

Auch wenn die Ermöglichung des Lernens Erwachsener bzw. die Politik zur Erwachsenenbildung in der international-vergleichenden Bildungsforschung bisher selten vertreten ist und sich auch die genuin international-vergleichende Erwachsenenbildungsforschung bislang nicht im Sinne eines systematischen Forschungsprogramms mit Bildungspolitik beschäftigt hat, liegen verschiedene internationale Einzelstudien vor, die sich mit Fragen der Bildungspolitik und des lebenslangen Lernens beschäftigen (für eine Übersicht siehe Field et al. 2016). So untersucht beispielsweise Schemmann (2007) die Bedeutung internationaler und supranationaler Organisationen für nationale Bildungspolitik oder Ioannidou (2010) die Bedeutung dieser Organisationen für das Konzept des Lebenslangen Lernens in drei exempla- 
rischen Staaten. Zur Erforschung bildungspolitischer Fragestellungen in einer international-vergleichenden Perspektive arbeitet die Erwachsenenbildungsforschung zunehmend interdisziplinär und mit Theorien aus Nachbardisziplinen wie der vergleichenden Politikwissenschaft, ${ }^{1}$ der Ökonomie oder der Soziologie (Hurrelmann et al. 2011; Graf 2016; Knauber und Ioannidou 2016). Erste Ansätze einer institutionellen Kooperation zwischen der Forschung zur Erwachsenenbildung und der politikwissenschaftlichen Forschung finden sich z. B. im Tübinger Promotionskolleg „International-vergleichende Forschung zu Bildung und Bildungspolitik im Wohlfahrtsstaat“" (Schmid et al. 2011, S. 7), das von 2009 bis 2017 von der BöcklerStiftung gefördert wurde.

Insgesamt rücken die bisherigen Beiträge der international-vergleichenden (Erwachsenen-) Bildungsforschung zur Bildungspolitik Fragen der Teilnahme, der Kompetenzen und der Steuerung in den Fokus (siehe z. B. oben, Boeren und Holford 2016; Martin und Rüber 2016); Fragen und theoretische Zugänge, die Bildung und Bildungspolitik breiter, z. B. auch mit Blick auf die gesellschaftliche und wohlfahrtsstaatliche Einbettung diskutieren, finden bislang jedoch noch kaum eine systematische Berücksichtigung.

Bislang werden solche Fragen vor allem mit Zugängen der vergleichenden Politikwissenschaft adressiert (Gift und Wibbels 2014; Jakobi et al. 2010; Schmid et al. 2011). ${ }^{2}$ Busemeyer und Trampusch (2011) zeichnen diese Entwicklung in ihrem Literatur-Review Comparative Political Science and the Study of Education nach und zeigen, dass in der vergleichenden Politikwissenschaft insbesondere Arbeiten zu Parteienpolitik bei Bildungsreformen, vergleichende politische Ökonomik zu Skill Formation, Zusammenhänge zwischen Bildung und Wohlfahrtsstaaten und die Internationalisierung von Bildung im Fokus der Forschung standen (ebd., S. 413 f.). Auch wenn Bildung als Thema in der vergleichenden Politikwissenschaft ein recht junges, gleichwohl aber etabliertes Forschungsfeld ist, lohnt sich eine genauere Betrachtung der Disziplin und ihrer international-vergleichenden Forschung zur Bildungspolitik. Dies liegt zum einen am Gegenstandsbereich der Bildungspolitik und dessen inhaltlicher Nähe zur Disziplin. Zum anderen bietet die Disziplin mit ihren Ansätzen und Theorien (insbesondere im Bereich der Policy-Forschung) einen fundierten Zugang zur Forschung über Bildungspolitik, der auch für die international-vergleichende Erwachsenenbildungsforschung anregend sein könnte. Dies betrifft z. B. die Erforschung jüngerer Entwicklungen der Internationalisierung und komplexer internationaler Interaktionsgeflechte, des Einflusses zivilgesellschaftlicher Akteure oder

\footnotetext{
1 Mit der vergleichenden Politikwissenschaft ist im Folgenden ein Teilgebiet der Politikwissenschaft gemeint, in dem Staats- und Regierungsformen (polity), Strukturen und Inhalte (policy) sowie Prozesse (politics) vergleichend betrachtet werden. Davon abgrenzen lässt sich (vergleichende) soziologische Forschung, die sich theoretisch und empirisch mit den Bedingungen und Formen des sozialen Verhaltens bzw. des gesellschaftlichen Zusammenlebens beschäftigt und deren Forschungen hier ausgeblendet bleiben.

2 Busemeyer und Trampusch differenzieren zwischen der vergleichenden Politikwissenschaft einerseits und der vergleichenden Forschung zu Wohlfahrtsstaaten andererseits. Während Erstere Bildung als Forschungsthema lange vernachlässigte, ist Bildung in Letzterer als Thema schon lange präsent (2011, S. 413). Im vorliegenden Beitrag werden unter vergleichender Politikwissenschaft beide Forschungsstränge mit einbezogen.
} 
der Formen und Effekte neuer Governance-Strukturen (Martens et al. 2007). So merkt Ioannidou bereits 2010 an, dass es sich

(...) als erforderlich erweisen [könne], Theorien aus Nachbardisziplinen zur Bearbeitung von Forschungsfragen heranzuziehen. Ansätze aus der vergleichenden Policy-Forschung können stimulierend auf die Erklärung von Rezeptionsund Implementationsphänomenen im Rahmen der international-vergleichenden Bildungsforschung wirken (Ioannidou 2010, S. 278).

Vor diesem Hintergrund wird in diesem Beitrag ein Literatur-Review zu Publikationen der vergangenen zehn Jahre dargestellt, die den Bereich der internationalvergleichenden Forschung über Bildungspolitik fokussieren und die mit Ansätzen und Theorien der vergleichenden Politikwissenschaft und insbesondere deren Teilgebiet der Policy-Forschung arbeiten. Den oben genannten Beitrag von Busemeyer und Trampusch (2011) ergänzend und erweiternd ist das Ziel des Beitrags, die verwendeten theoretischen Zugänge aufzuzeigen, kritisch zu würdigen und über die Ergebnisse der Forschung einen thematischen Überblick zu geben. Der Beitrag adressiert dabei Forschende der international-vergleichenden (Erwachsenen-)Bildungsforschung, für die er beleuchtet, welche Theorien und Ansätze der vergleichenden Politikwissenschaft sich zur Erforschung welcher Fragestellungen der international-vergleichenden Erwachsenenbildungsforschung eignen.

Der vorliegende Beitrag ist bildungsbereichsübergreifend angelegt und schließt schulische (ab Sekundarbereich), berufliche, universitäre Bildung und Erwachsenenbildung sowie umfassendere Konzepte wie das Konzept des Lifelong Learning (LLL) gleichermaßen ein. Dies liegt zum einen daran, dass die Forschungspublikationen, die mit politikwissenschaftlichen Ansätzen und Theorien arbeiten, (bisher) selten das Thema Erwachsenenbildung im Blick haben. Zum anderen bzw. ergänzend werden Erträge sowie Desiderate und damit das Potenzial politikwissenschaftlicher Bildungsforschung für die international-vergleichende Erwachsenenbildungsforschung erst im Vergleich mit anderen Bildungsbereichen sichtbar.

Im Folgenden wird zunächst die systematische Literaturrecherche nachgezeichnet. Danach folgt die Darstellung der Literatur nach den in ihr verwendeten Ansätzen und Theorien der Policy-Forschung sowie ein Überblick über deren Ergebnisse. Abschließend erfolgt ein Ausblick mit möglichen Anknüpfungspunkten für die international-vergleichende Erwachsenenbildungsforschung.

\section{Systematische Recherche von Literatur}

Diesem Beitrag liegt eine systematische Literatur-Review zugrunde (Jesson et al. 2011; Petticrew und Roberts 2006). Die Suche wurde aufgrund der Menge an Veröffentlichungen auf die Jahre 2006 bis 2016 begrenzt. Die Recherche wurde im einschlägigen sozialwissenschaftlichen Rechercheportal sowiport, ${ }^{3}$ welches eine Metarecherche in unterschiedlichen Datenbanken ermöglicht, in der Datenbank der Cambridge University Press sowie in Fachzeitschriften, die für den genannten Zeit-

\footnotetext{
3 Siehe auch http://sowiport.gesis.org/Search/Advanced.
} 
raum vollständig gesichtet wurden, durchgeführt. Für soziologische und politikwissenschaftliche Publikationsmedien wurden die 50 höchstgerankten Zeitschriften der Fachdisziplin im Scimago Journal \& Country Rank (Stand Dezember 2016) nach Journals durchgesehen, in deren thematisches Spektrum Publikationen über Bildung bzw. Bildungspolitik passen und diese dann gesichtet. Des Weiteren wurden die entsprechenden Jahrgänge der Zeitschriften Comparative Education Review, Compare: A Journal of Comparative and International Education, Zeitschrift für Erziehungswissenschaft, Zeitschrift für Pädagogik und Zeitschrift für Weiterbildungsforschung Report durchgesehen. In der Metadatenbank sowiport wurde nach den Suchbegriffen „education policy“, ,,education politics“, ,,education polity“ bzw. „Bildungspolitik“ gesucht. In politikwissenschaftlichen und soziologischen Zeitschriften wurde nach dem Suchbegriff ,education“ bzw. „Bildung“ gesucht. Die Recherche wurde bis Januar 2017 durchgeführt. Zunächst wurden Titel und (wenn vorhanden) Abstracts der Beiträge hinsichtlich der Anschlussfähigkeit an den thematischen Fokus dieses Literatur-Reviews gesichtet. Kriterien der Auswahl waren der Zeitraum der Veröffentlichung zwischen 2006 und 2016, der Einbezug empirischer Daten, der Vergleich von mindestens zwei Ländern, und Forschung, die sich auf westlich entwickelte Industriestaaten bezieht. Nach dieser Vorauswahl wurden die dokumentierten 120 Beiträge genauer hinsichtlich theoretischer Bezüge überprüft. Zum Schluss flossen 65 Titel in diese Literatur-Review ein. Bei der überwiegenden Zahl der Publikationen handelt es sich um Journalbeiträge (48). Monografien (7) und Beiträge in Sammelbänden (10) bildeten eine Minderheit. Methodisch arbeitet die Hälfte der Beiträge mit quantitativen Methoden (28), knapp 25 Beiträge arbeiten qualitativ (z. B. mit Experteninterviews, Dokumenten- und Literaturanalysen) und knapp 12 Beiträge arbeiten mit einer Mischung aus quantitativen und qualitativen Methoden.

\section{Auswertung der ausgewählten Literatur nach Ansätzen und Theorien der Politikwissenschaft}

Für diese Literatur-Review wurden international-vergleichende Beiträge mit bildungspolitischen Themen ausgewählt, die Ansätze und Theorien der vergleichenden Politikwissenschaft (im weiteren Sinne ${ }^{4}$ ) verwenden, um ihre Länderauswahl $\mathrm{zu}$ begründen, ihre Hypothesen zu bilden und ihre Ergebnisse einzuordnen und zu interpretieren. Nicht alle politikwissenschaftlichen Theorien und Ansätze eignen sich zur Erforschung bildungspolitischer Fragestellungen. Im Folgenden werden die Ansätze vorgestellt, die in den ausgewählten Beiträgen gefunden wurden und fast alle der Policy-Forschung zuzuordnen sind (Wenzelburger und Zohlnhöfer

\footnotetext{
${ }^{4}$ Damit ist gemeint, dass es in einzelnen Fällen zu Überschneidungen mit soziologischen und ökonomischen Theorien und Ansätzen kommt, da diese im multidisziplinären Feld der Bildungspolitik nicht immer trennscharf sind.
} 
2015). ${ }^{5}$ Auch handelt es sich nicht immer um Theorien (im Sinne von expliziten und empirisch eindeutig verankerten Modellen), aus denen Hypothesen abgeleitet werden können, sondern teilweise um umfassende Analyserahmen (wie bspw. den Akteurzentrierten Institutionalismus). Letzteres erklärt, warum Autoren zum Teil mehrere der unten ausgeführten theoretischen Ansätze für ihre Arbeiten verwenden. Für die Erfassung unterschiedlicher bildungspolitischer Staatstätigkeit wird in den ausgewählten Texten berücksichtigt, inwieweit die Länder sich in ihrer Sozialpolitik unterscheiden (3.1 Wohlfahrtstaaten), über unterschiedliche Produktionsregime verfügen (3.2 Varieties of Capitalism), von bestimmten Parteien (mit-)regiert werden (3.3 Parteiendifferenztheorie), in unterschiedlichem Maß Globalisierungs- und Europäisierungstendenzen ausgesetzt sind (3.4 Globalisierung und Europäisierung), Pfadabhängigkeit zeigen (3.5 Pfadabhängigkeit) und eine bestimmte Struktur von Interessengruppen zeigen (3.6 Akteurzentrierter Institutionalismus, AZI). Anzumerken ist, dass Educational Governance nicht als eigener Ansatz berücksichtigt wird, da es sich um eine Forschungsperspektive handelt und nicht um einen konkreten Ansatz oder eine Theorie. Institutionalistische Ansätze in ihren vielfältigen Formen konnten aus Platzgründen nur einbezogen werden, wenn sie mit Pfadabhängigkeit und dem AZI arbeiten. Innerhalb der Kapitel werden die Beiträge danach sortiert, ob sie sich mit dem bildungspolitischen Input bzw. dem Willensbildungs- und Entscheidungsprozess der Politikformulierung, dem Output bzw. dem Politikergebnis (Gesetze, Beschlüsse, z. B. Bildungsausgaben) oder dem Outcome bzw. den Wirkungen politischer Entscheidungen (z. B. Bildungschancen) beschäftigen.

\subsection{Wohlfahrtsstaatentheorie}

Die Wohlfahrtsstaatentheorie nach Esping-Andersen (1998) geht davon aus, dass Entscheidungen über die Organisation und Ausgestaltung des öffentlichen Sektors und wohlfahrtsstaatliche Steuerung Auswirkungen auf soziale Segregation haben. Esping-Andersen geht mit seinem Cluster von Regime-Typen entlang der beiden Dimensionen Dekommodifizierung ${ }^{6}$ und Stratifizierung ${ }^{7}$ auf Unterschiede zwischen Sozialstaaten bezüglich des Verhältnisses von Markt und Staat und insbesondere

\footnotetext{
${ }^{5}$ Die Policy-Forschung beschäftigt sich mit der Beschreibung, Analyse und Erklärung von Gemeinsamkeiten und Unterschieden der Staatstätigkeit zwischen Staaten in einzelnen Politikfeldern, wie bspw. auch der Bildungspolitik. Mit Staatstätigkeit sind Politiken gemeint, die in der Regel von staatlichen Akteuren bzw. Regierungen beschlossen werden, aber auch Politiken unabhängiger Institutionen (Wenzelburger und Zohlnhöfer 2015), wie Zentralbanken, Regierungsbehörden und Interessengruppen wie Sozialpartner oder Bildungsverbände.

${ }^{6}$ Als Dekommodifizierung wird die relative Unabhängigkeit des Individuums vom Arbeitsmarkt bezeichnet (Esping-Andersen 1998, S. 21 f.). Ein hoher Grad an Dekommodifizierung deutet auf einen großzügigen Sozialstaat hin; das Individuum ist nicht so stark gezwungen, die Arbeitskraft als Ware auf dem Markt anzubieten (ebd., S. 37).

7 Die Stratifizierung zeigt, inwieweit ein Wohlfahrtsstaat nicht nur Ungleichheiten abbaut, sondern diese selbst erzeugt oder zementiert: „Apart from its purely income-distributive role, the welfare state shapes class and status in a variety of ways. The education system is an obvious and much-studied instance, in which individuals' mobility chances not only are affected, but from which entire class structures evolve" (ebd., S. 57 f.).
} 
auf die Ausprägung von Sozialleistungen ein. So differenziert er zwischen drei idealtypischen Regimen:

1. dem liberalen Wohlfahrtstaat mit der Betonung des freien Marktes (geringe Dekommodifizierung und niedrige institutionelle Stratifizierung) in einem begrenzt ausgebauten Sozialstaat (USA, CAN, AU);

2. dem konservativen Wohlfahrtsstaat mit korporatistischen Strukturen (mittlere Dekommodifizierung, hohe Stratifizierung) (AT, FR, DE) und

3. dem sozialdemokratischen Wohlfahrtsstaat, der individuelle Unabhängigkeit vom Markt ermöglicht (hohe Dekommodifizierung, welche zu einer verringerten sozialen Ungleichheit führt (ebd., S. 26 ff.) und geringe Stratifizierung) (skandinavische Länder).

Die Theorie wurde, auch von Esping-Andersen selbst, mehrfach erweitert und modifiziert, beispielsweise um einen mediterranen Typ oder ein postsozialistisches Regime. Eine Zuordnung der Länder zu den Regimen, wie auch die Regimetypen selbst, sind in der Literatur bis heute nicht einheitlich. Die Verbindung von Bildungspolitik zur Wohlfahrtsstaatentheorie wird von den in dieser Review ausgewählten Autoren insofern hergestellt, als dass Bildung und der Zugang zu Bildung als integraler Aspekt des umverteilenden Sozialstaats, der Wohlfahrtspolitik sowie als Erweiterung von Politiken sozialer Absicherung gesehen wird (Heidenheimer 1981). ${ }^{8}$ Die Auswirkungen von Umverteilung im Bildungsbereich sind jedoch wesentlich komplexer als in anderen Bereichen des Sozialstaats (Busemeyer 2015). ${ }^{9}$ Um Differenzen zwischen Staaten in den politischen Inhalten (Output, z. B. Bildungspolitik) und dem Ergebnis bzw. der Wirkung von Politik (Outcome, z. B. Bildungsteilnahme, Bildungschancen) zu erklären, rücken zunehmend Merkmale auf der Makroebene ins Blickfeld. Gruppierungen bzw. Regimetypen von Staaten mit ähnlichen Merkmalen der Makroebene ähneln sich in Systemvoraussetzungen und Opportunitätsstrukturen (Kaufmann et al. 2014, S. 39). Die gefundenen Beiträge, die die Wohlfahrtsstaatentheorie nutzen, arbeiten vorwiegend mit quantitativen Analysen vorhandener (meist international-vergleichender) Datensätze, wie z. B. von der OECD (Education at a Glance-Datenbank, PISA), dem International Social Survey Programme (ISSP), dem Eurobarometer, dem EU-LFS etc., größeren Länderfallzahlen und fokussieren den Output und Outcome von Bildungspolitik. Thematisch geht es in den Beiträgen beispielsweise um den Einfluss des Wohlfahrtsstaatsregimes auf Schul- und Hochschulpolitik (Pechar und Andres 2011; Willemse und de Beer 2012), Beteiligung an betrieblicher Weiterbildung (Markowitsch et al. 2013), LLL (Rubenson 2006) und um die Verbindung zwischen Präferenzen auf der Makroebene (Wohlfahrtsstaaten) und individueller Ebene (Busemeyer 2013).

Den Output von Bildungspolitik in Form von Bildungsausgaben in den Blick nehmend, folgert Hega (2011) nach einer Analyse von OECD-Daten und dem Einbezug zahlreicher Vorarbeiten, dass sich konservative und liberale Regime in ihren

\footnotetext{
8 Auch in die andere Wirkungsrichtung beeinflussen Arbeitsmarkt und wohlfahrtsstaatliche Institutionen die Bildungspolitik.

9 Dies geschieht in Abgrenzung zu Wilensky, nach dem sich die Bildungspolitik von anderen Sozialpolitiken unterscheidet (,education is special“, Wilensky 1975, S. 3).
} 
Sozialpolitiken und Bildungssystemen ähneln. Er stellt jedoch fest, dass liberale Regime mehr für Bildung ausgeben (mit hohem Anteil privater Bildungsausgaben) als andere Regime, wobei dieses Geld vor allem in den Schulsektor und den Hochschulbereich fließt. Konservative Regime geben hingegen weniger für Bildung aus; diese Ausgaben fließen zu großen Anteilen in berufliche und Hochschulbildung. Sozialdemokratische Regime haben den höchsten Anteil staatlicher Bildungsausgaben; Ausgaben fließen vor allem in Schule und Hochschule. Willemse und de Beer (2012) clustern Staaten anhand einer quantitativen Analyse verschiedener Datensätze nach der institutionellen Ausgestaltung von Hochschulpolitik und stellen fest, dass ihre vorgefundene Clusterung grob der Zuordnung von Staaten zu den drei Wohlfahrtsregimen entspricht; bei liberalen und konservativen Staaten die jeweils zugeschriebene Ausprägung von Dekommodifizierung und Stratifizierung jedoch nicht mit den Erwartungen übereinstimmt. Eine Ausdifferenzierung und Modifikation des Wohlfahrtstaatenansatzes findet sich bei der Untersuchung der Verankerung von inklusiver Bildung von Arnesen und Lundahl (2006), die eine genauere Beschreibung der Bildungspolitik in sozialdemokratischen und liberalen Regimen auf Grundlage der Analyse nationaler Statistiken vornehmen. In dieser wird auf die politische Funktion von Bildung, deren Wertehintergrund und auf politikinitiierende Akteure eingegangen.

Die Verbindung von bildungspolitischem Output und Outcome fokussierend, führen Hurrlemann et al. (2011) die Bildungspolitik als elementaren Bestandteil von Wohlfahrtsstaaten ein, der soziale und wirtschaftliche Entwicklungschancen und die Teilhabe erhöht. Die quantitativen Analysen von OECD-Daten zeigen, dass Sozialund Bildungspolitik sich ergänzen können, um positive Bildungsbilanzen (und damit die längerfristige Wohlfahrt eines Staates) zu schaffen und Bildungsgleichheit durch eine gleichmäßige Investition in alle Bildungsbereiche erreicht wird. Busemeyer (2013, 2014) beschäftigt sich mit der Verbindung zwischen Präferenzen auf der Makroebene (Wohlfahrtsstaaten) und individueller Ebene. Er zeigt mit quantitativen Analysen von Daten der OECD und des ISSP sowie bezugnehmend auf qualitative Daten, dass bei hohen privaten Bildungsausgaben (liberale Regime) die individuelle Unterstützung für Umverteilung geringer ist. In Ländern mit höheren Anteilen öffentlicher Bildungsausgaben (sozialdemokratische Regime) wird Umverteilung von Individuen eher unterstützt.

Beiträge, die primär den Outcome von Bildungspolitik in den Blick nehmen, beschäftigen sich mit Bildungsstratifizierung, Bildungschancen und -teilnahme. Eine Überprüfung von Bildungs- und Absicherungspolitiken und deren Einfluss auf soziale Stratifizierung mit einer quantitativen Analyse von OECD-Daten zeigt, dass verschiedene bildungspolitische Herangehensweisen in zentraleuropäischen Staaten (starke Stratifizierung) im Vergleich zu liberalen Staaten (stärkere Angleichung) und den skandinavischen und südeuropäischen Staaten (Stratifizierung bezüglich Renten; Angleichung in der Bildung) sichtbar werden (Beblavý et al. 2013). Sich ausschließlich auf den Bildungsbereich beziehend, untersucht Busemeyer (2014) anhand einer Analyse von OECD-Daten in mehreren Staaten die Bildungsstratifizierung und identifiziert drei Cluster, die denen der Wohlfahrtsstaaten ähneln: Ein liberales Cluster mit geringer Dekommodifizierung (hoher Anteil privater Bildungsausgaben) und Stratifizierung (USA, CAN, UK), ein Cluster mit geringer Bildungsstratifizierung 
und hoher Dekommodifizierung (skandinavische Staaten, NL, FR) und das zentraleuropäische Cluster mit hoher Bildungsstratifizierung und mittlerer Dekommodifizierung (DE, AT, CH). Pechar und Andres (2011) betrachten marktorientierte Reformen der Schul- und Hochschulpolitik in den USA und Schweden. Das Ergebnis ihrer quantitativen Analyse von OECD-Daten zeigt Zielkonflikte (trade-offs): Liberale Regime bieten Chancen für die Individuen, die den Anforderungen der Wissensgesellschaft entsprechen, während die, die diesen Anforderungen nicht entsprechen (Schulabbrecher; Personen, die keine akademische Ausbildung anstreben) vom System benachteiligt werden. In konservativen Regimen zeigen sich bessere Chancen für Personen, die keine akademische Bildung anstreben, da es ein umfassendes berufliches Bildungssystem gibt - jedoch wird die soziale Ungleichheit zementiert. In sozialdemokratischen Regimen werden den Ergebnissen nach einige der Zielkonflikte vermieden, so gibt es einen deutlichen Widerstand gegen die Einführung von Studiengebühren und wesentlich höhere Ausgaben für Hochschulbildung als in konservativen Regimen. Mit Bildungsteilnahme und Bildungschancen der erwerbsfähigen Bevölkerung beschäftigen sich Martin und Rüber (2016) anhand einer quantitativen Analyse von Daten des EU-LFS. Sie stellen wie Rubenson und Desjardins (2009) fest, dass die Ungleichheit der Bildungschancen in skandinavischen (und eher sozialdemokratisch geprägten) Staaten am geringsten ist.

Beispiele für die Weiterentwicklung von wohlfahrtsstaatlichen Typologien für Bildung auf Grundlage quantitativer Analysen von (meist) OECD-Daten sind Arbeiten von Iversen und Stephens (2008), die herausarbeiten, dass sich die Beziehungen zwischen Sozialversicherung, Kompetenzentwicklung und öffentlichen Bildungsausgaben gegenseitig verstärken. Sie leiten daraus drei unterschiedliche Welten der Humankapitalbildung ab, die historisch in der Ausgestaltung des wirtschaftlichen Systems, des Wahlsystems und des Parteienwettbewerbs begründet sind. Green et al. (2006) entwickeln eine Typologie auf Grundlage von Wohlfahrtsstaaten, Arbeitsmarkts- und LLL-Systemen und unterscheiden zwischen drei Typen von Wissensökonomien: liberale Regime, Regime der sozialen Marktwirtschaft und sozialdemokratische Regime. Desjardins (2013) entwickelt aufbauend auf der Wohlfahrtsstaatentheorie und den Varianten des Kapitalismus eine Kategorisierung von Regimen, die das Verhältnis zwischen Markt und anderen koordinierenden Institutionen zum Bildungssystem eines Landes einordnet. Markowitsch et al. (2013) beschreiben bei einer Untersuchung der Beteiligung an betrieblicher (Weiter-)Bildung als Teil des LLL und der institutionellen Ausgestaltung der betrieblichen Bildung in verschiedenen Ländern fünf Typen: sozialdemokratischer, konservativer, ,familaristic/subprotective", liberaler und neoliberaler bzw. neokonservativer Wohlfahrtsstaat.

Zusammenfassend lässt sich festhalten, dass eine Verbindung von Bildungspolitik und Wohlfahrtsstaatentheorie von den Autoren erst argumentativ geschaffen werden muss, da diese nicht im Ansatz integriert ist. Deutliche Ähnlichkeiten zwischen den Regimetypen der Theorie und den Länderclustern lassen sich als Ergebnis empirischer Untersuchungen zu Bildung finden. Einige Autoren modifizieren die Regimetypen und differenzieren diese weiter im Hinblick auf einzelne Bildungsbereiche aus. Ein Schwerpunkt der jüngeren Forschung zeigt sich in der Verwendung der Theorie zum Vergleich von bildungspolitischen Outcomes zwischen Staaten. 


\subsection{Varieties of Capitalism (VoC)}

Die Spielarten des Kapitalismus bezeichnen einen Theoriebestand, der Länder nach der Koordination des Wirtschaftsgeschehens differenziert (Höpner 2015) und davon ausgeht, dass es komplementäre Beziehungen zwischen Institutionen gibt (Hall und Soskice 2001, S. 18). Hall und Soskice differenzieren zwischen koordinierten (DE, AT), CME und liberalen Marktökonomien (USA, UK), LME. ${ }^{10}$ Auch wenn einzelne Publikationen den Ansatz nur als Heuristik der Länderauswahl nutzen (SoutoOtero und Ure 2012), ist für die meisten der ausgewählten Publikationen die Unterscheidung zwischen koordinierten und liberalen Ökonomien bezüglich des Ausbildungswesens entscheidend. Nach Estevez-Abé et al. (2001) bringen unterschiedliche Produktionsstrategien unterschiedliche Qualifikationsanforderungen mit sich. Je nach Bedarf an spezifischen oder allgemeinen Qualifikationen sind Ausbildungssysteme von Ökonomien gestaltet: In koordinierten Ökonomien werden die für die Arbeit notwendigen spezifischen Fähigkeiten und Kompetenzen in korporatistischen Strukturen in den Unternehmen selbst und unter der Regulation von Sozialpartnern hervorgebracht. In liberalen Ökonomien hingegen findet die (eher allgemeine) und marktgesteuerte Ausbildung in der Regel außerhalb von Unternehmen in schulischen Ausbildungsgängen oder Universitäten statt (Culpepper und Thelen 2008). Ähnlich wie bei der Wohlfahrtsstaatentheorie liegt der Schwerpunkt der ausgewählten Beiträge des VoC-Ansatzes auf den bildungspolitischen Outputs und Outcomes. Methodisch sind die Beiträge vielseitiger. So zeigen sich neben Beiträgen, die nationale und international-vergleichende Datensätze für kleinere Länderfallzahlen quantitativ im Hinblick auf Wirkung spezifischer Variablen auf politischen Output und Outcome auswerten, auch einzelne Arbeiten, die qualitative Forschungsdesigns haben (bspw. Experteninterviews, Dokumentenanalysen).

Den politischen Outcome in Form von Kompetenz- und Bildungsspezifität in CME und LME fokussierend beschäftigen sich Roosmaa und Saar (2012) mit der Weiterbildungsselektivität in EU-Staaten. Sie zeigen anhand einer Analyse von AES-Daten, dass diese (sowie die Weiterbildungsteilnahme Geringqualifizierter) eher durch die Nachfrageseite des Arbeitsmarktes (Qualifikationsanforderungen) und nicht durch die Qualifikationsstruktur der Erwerbstätigen erklärt wird. Die Kompetenzspezifität an Hochschulen in den Blick nehmend, untersucht Hoelscher (2012) die Länder England (LME) und Deutschland (CME) auf Grundlage einer quantitativen Analyse von Daten der REFLEX-Studie mit dem Ergebnis, dass, wie der VoCAnsatz vermuten lässt, deutsche Hochschulabsolventen stärker spezifische, britische eher generelle Kompetenzen betonen und die Passung zwischen Studium und Beruf in Deutschland enger ist. Vogtenhuber (2015) beschäftigt sich mit der Aufteilung der Schüler im Sekundarschulbereich und deren Auswirkung in Form von Einfluss auf die berufliche Weiterbildung und ihren Ertrag. Die Ergebnisse der quantitativen Analyse verschiedener Datensätze zeigen, dass (trotz der Variation länderübergreifender Weiterbildungsintensität nach der Bildungsbeteiligung und den Bildungsausgaben)

\footnotetext{
10 Einen eigenen Typus bilden die Länder des Mittelmeerraums (Frankreich, Italien, Spanien, Portugal, Griechenland, Türkei) mit einzelnen Eigenschaften, die sich sowohl mit CME als auch LME überschneiden.
} 
die Erträge der Weiterbildung stark mit der Qualität und dem Aufteilungszeitpunkt der weiterführenden Schulen verbunden sind. Dieser Befund deutet darauf hin, dass das Weiterbildungsniveau mit dem Qualifikationsniveau in Bezug auf Qualifikationsnachfrage und Bildungsausgaben zusammenhängt. In Systemen, in denen Schulabgänger und Beschäftigte tatsächlich über höhere Qualifikationen verfügen, ist hingegen der Produktivitätszuwachs durch Aus- und Weiterbildung größer. Ebenfalls mit der Aufteilung der Schülerschaft in der Sekundarstufe beschäftigt sich Baldi (2012), jedoch in Bezug auf die Gründe, warum sich in Deutschland und England verschiedene Systeme ausbildeten. Er führt dies auf die Verknüpfung von Bildung und Wirtschaft zurück, wonach in CME Schüler früher und in LME eher später aufgeteilt werden. Die Ergebnisse einer rekonstruktiven qualitativen Analyse zeigen jedoch, dass nicht nur wirtschaftliche Akteure in den beiden Ländern Einfluss auf diese Ausgestaltung des Systems nahmen, sondern auch politische Akteure und Strukturen.

Thematisch breiter angelegt als die bisher beschriebenen Arbeiten und eher auf den politischen Output fokussiert, ist die Untersuchung von Bosch und Charest (2008), die formale Bildung in CME (DE, DK) und LME (USA, CAN) und Südkorea (mit einer noch stärker akademisierten Bildung als die beiden untersuchten LME-Staaten) anhand der deskriptiven Auswertung unterschiedlicher Datensätze und Literatur. Die Ergebnisse zeigen, dass sich alle drei Typen im Bereich der allgemeinen Bildung angleichen, wird doch versucht, diese insgesamt anzuheben. Auch bei der akademischen Bildung zeigen sich Konvergenzen in deren Expansion. Bei der beruflichen Bildung unterscheiden sich die Systeme weiter grundlegend; auch innerhalb der CME sehen die Autoren deutliche Unterschiede in der beruflichen Ausbildung. Um die beruflichen Ausbildungsformen in CME (DE) und LME (USA) besser unterscheiden zu können, berücksichtigt Graf (2016) zusätzlich die Ausprägung von Stratifizierung und Standardisierung der Berufsbildung, die Steuerung durch Sozialpartner, Kooperationen von Arbeitgebern und Finanzierung. Der Autor kann mithilfe von Experteninterviews und Dokumentenanalysen zeigen, dass es große Überschneidungen zwischen hochschulbasierten Formaten in Deutschland und den USA gibt (duales Studium), während sich die klassische duale Lehre und das duale Studium innerhalb Deutschlands deutlich unterscheiden.

Eine Reihe von Veröffentlichungen befasst sich ausschließlich mit CME und der Herausbildung beruflich und akademisch qualifizierender Bildungssysteme (Skill Formation). Die Arbeiten nehmen dabei primär bildungspolitische Outputs und Outcomes in den Fokus, wobei eine Differenzierung zwischen beiden nicht trennscharf verläuft. Die Kompetenz- und Bildungsspezifität vom CME untersuchend, stellt Jensen (2011) mit einer quantitativen Analyse verschiedener Datensätze fest, dass die Deindustrialisierung eine bedeutende Bedrohung für Arbeitnehmer ist, insbesondere in Ländern, in denen die Kompetenz-/Bildungsspezifität hoch ist. Busemeyer (2009) kritisiert ebenfalls bezugnehmend auf die Kompetenz- und Bildungsspezifität innerhalb von CME nach Durchsicht bisheriger Berechnungen und Literatur, dass die Variation innerhalb der CME nicht hinreichend erfasst wird, weshalb er eine eigene Differenzierung nach zwei Dimensionen vorschlägt: der Grad der Unternehmensbeteiligung in der Bildungsausgestaltung und der Grad der Zertifizierung von beruflichen Fähigkeiten durch das Bildungssystem. Dadurch ergeben 
sich für den Autor drei unterschiedliche Bildungsregime: das segmentierte (unternehmensbasierte) Regime in Japan, das integrative (schulbasierte berufliche) Regime in Schweden und das differenzierte (berufliche und praxisbezogene) Regime in Deutschland. Culpepper (2007) untersucht die Wechselwirkungen zwischen allgemeiner und spezifischer (Berufs-)bildung und Arbeitgebern in der Schweiz und Österreich seit dem Zweiten Weltkrieg quantitativ. Er kann zeigen, dass trotz ähnlicher Startbedingungen die Schweiz ein eher allgemeines (Berufs-)Bildungssystem ausbildete, während das System in Österreich spezifischer ist. Dies führt er auf die unterschiedliche Dominanz von großen und kleine und mittlere Unternehmen (KMU) zurück: In der Schweiz dominieren größere, wenig kostensensible Arbeitgeber, die durch eine Orientierung am internationalen Markt einen größeren Nutzen allgemeiner Fähigkeiten ihrer Belegschaft sehen. In Österreich wiederum dominieren kleinere Arbeitgeber mit spezifischen Bildungsinteressen. Ebner und Nikolai (2010) vergleichen ebenfalls Österreich, die Schweiz und zusätzlich Deutschland in Bezug auf ihre berufliche Ausbildung mit einer Übersicht an statistischen Daten der Länder, politischen Dokumenten und Literatur. Während in den letzten beiden Staaten weiter das duale Ausbildungssystem dominiert, hat sich in Österreich neben diesem System ein schulisches Berufsbildungssystem ausgebildet, was die Autoren mit der Dominanz der Sozialdemokratie (und Kooperation mit Christdemokraten), der Steuerung des Bildungswesens durch den Zentralstaat und der Unterstützung der Entwicklung durch Arbeitgeber und Gewerkschaften begründen. Einen Zusammenhang (Culpepper 2007) zwischen Unternehmensinteressen und beruflicher Bildung (und deren Verknüpfung mit Hochschulbildung) in den CME Deutschland, Österreich und Schweiz zeigen Nikolai und Ebner (2013). In allen Ländern gibt es eine Verknüpfung von beruflicher Bildung und Hochschule: doppelqualifizierende Abschlüsse ( $\mathrm{CH}, \mathrm{AT})$, Anrechnung beruflicher Kompetenzen beim Hochschulzugang (DE). Die Interessen von Unternehmen spalten sich jedoch nicht nach Unternehmensgröße, sondern hängen von der Einbettung des Berufsbildungssystems in das Gesamtbildungssystem, der Vorbildung von Azubis sowie der Attraktivität des Berufsbildungssystems im Vergleich zu alternativen Bildungsgängen ab. Graf (2013) betrachtet ebenfalls die oben genannten Länder, die in der Vergangenheit eine Kluft zwischen beruflicher Ausbildung und Hochschulbildung hatten, und untersucht die neu entwickelten ,hybriden“ Systeme der Länder, die eine Mischung beider Bereiche erreichen sollen: duale Studiengänge in Deutschland, berufsbildende höhere Schulen in Österreich und doppelqualifizierende Ausbildungsabschlüsse in der Schweiz. Im Gegensatz zu den vorherigen Autoren nutzt er dafür jedoch ein qualitatives Forschungsdesign mit Experteninterviews und Dokumentenanalysen. Er stellt fest, dass die neuen Systeme nicht zu einer Überwindung der Kluft, sondern zu einem Zustand der Koexistenz traditioneller und hybrider Systeme führen. Mit Bildung als sozialer Absicherung (insbesondere vor Arbeitslosigkeit) in CME beschäftigt sich Trampusch (2010) anhand einer historischen Analyse von Entwicklungen in Dänemark, der Niederlande und der Schweiz zwischen 1870 und 1940. In den CMEs entwickelten sich spezifische Bildungssysteme und ein umverteilender Sozialstaat mit sozialen Absicherungssystemen, auf welche die Arbeitgeber großen Einfluss nahmen. Sie weist aber darauf hin, dass Entwicklungen von sozialer Absicherung auch durch Gewerkschaften, das Arbeitgeber-Arbeitnehmer-Verhältnis, staatliche Akteure (und 
den staatlichen Eingriffen in den Arbeitsmarkt) sowie Industrialisierung beeinflusst wurden.

Zusammenfassend ist festzuhalten, dass es zum einen Beiträge gibt, die sich mit der Spezifität von Bildung in unterschiedlichen Regimen bzw. Staaten beschäftigen, und Beiträge, die die Ausbildung unterschiedlicher Bildungssysteme nachzeichnen (Skill Formation), wobei hier insbesondere die CME im Fokus stehen. Ein zentraler Bestandteil des VoC-Ansatzes, der in zahlreichen Arbeiten aufgegriffen wird, ist der Einfluss nicht-staatlicher wirtschaftlicher Akteure auf bzw. die Verknüpfung wirtschaftlicher Akteure mit Bildungspolitik und -system. Thematisch greifen die Beiträge den bildungspolitischen Output (Ausgestaltung Bildungsbereiche) und Outcome (Weiterbildungsselektivität) auf.

\subsection{Parteiendifferenztheorie}

Der Ansatz zu Parteien bzw. Parteiendifferenzen (Partisan Theory) geht davon aus, dass die Politik und damit auch die Bildungspolitik eines Landes davon beeinflusst wird, welche Partei oder Parteienkoalition die Regierung stellt (für einen Überblick siehe Wenzelburger 2015). Die Motive für die Durchsetzung von parteilich-gefärbten Politiken lassen sich in den Zielen der Wiederwahl/dem Ausbau von Wählerstimmen (vote-seeking) und der Durchsetzung präferierter politischer Inhalte (policy-seeking) finden (von Beyme 2000, S. 25). Ansätze, die in Verbindung mit Parteiendifferenzen gesehen werden, können die institutionellen Umstände des politischen Systems betreffen, wie beispielsweise Vetospieler/Vetopunkte. ${ }^{11}$ Zur Differenzierung von Parteipolitiken gibt es zwei Herangehensweisen: die Unterscheidung von Parteienfamilien einerseits und die Unterscheidung von Parteipositionen zu einzelnen Themen andererseits (Wenzelburger 2015, S. 92 ff.). Wie bei den bisher beschriebenen Ansätzen liegt der Schwerpunkt der Beiträge, die mit der Parteiendifferenztheorie arbeiten, auf bildungspolitischen Outputs. Als Output wird insbesondere die unterschiedliche Höhe öffentlicher und privater Bildungsausgaben verschiedener Staaten in den Blick genommen und deren Zusammenhang mit Regierungsparteien. Die Beiträge arbeiten methodisch vorwiegend mit quantitativen Längsschnittvergleichen internationalvergleichender Datensätze. Einige Beiträge beziehen sich auf qualitative Analysen des Manifesto-Projekt-Datensatzes. ${ }^{12}$ Dieser beinhaltet inhaltsanalytisch ausgewertete Parteiprogramme von über 50 Ländern von 1945 bis heute und erlaubt somit die Analyse ähnlich großer Länderfallzahlen im Längsschnitt wie die quantitativen Untersuchungen.

Nikolai (2007) kann mit einer kombinierten Längs- und Querschnittsanalyse in 28 OECD-Staaten von 1990 bis 2002 zeigen, dass sich die Regierungsbeteiligung von Linksparteien und liberalen Parteien positiv auf öffentliche Bildungsausgaben auswirkt, während säkular-konservative und christdemokratische Parteien eher einen

\footnotetext{
11 Dieser Ansatz geht davon aus, dass politische Entscheidungen entlang einer Entscheidungskette verlaufen, die Veto-Möglichkeiten bzw. Veto-Punkte eröffnet, an denen, bei einer Mobilisierung von Macht und Interessen, Einfluss auf den politischen Prozess und die Ergebnisse genommen werden kann (Tsebelis 1995).

12 Siehe auch: https://manifesto-project.wzb.eu (abgerufen am 08.06.2017).
} 
negativen Effekt zeigen. Dies könnte daran liegen, dass die ersten beiden Parteien eher soziale Umverteilung fördern möchten, um ein spezifisches Wählerklientel anzusprechen. Busemeyer et al. (2013) kommen mit ihrer qualitativen Untersuchung von Parteiprogrammen danach, wie sehr sie das Thema Bildungsausgaben betonen, zu ähnlichen Ergebnissen. Sie zeigen, dass die Bereitschaft, Bildungsausgaben zu erhöhen, zunehmend von sozialdemokratischen und liberalen Parteien vertreten wird, wobei dies kein einheitlicher Trend ist und eine große Variation zwischen den Ländern und über die Zeit besteht. ${ }^{13}$ Dies widerspricht Ergebnissen einer früheren Untersuchung des gleichen Datensatzes zu Parteiprogrammen von Jakobi (2011), die feststellte, dass die Differenzen zwischen Parteien zur Steigerung von Bildungsausgaben immer mehr abnehmen und sich ab den 1980er-Jahren praktisch keine Unterschiede mehr zwischen den Parteien finden lassen. Ansell (2010) bestätigt dies zunächst, kann jedoch zeigen, dass der ideologische Stand einer Partei ein besserer Prädiktor für die tatsächlichen Bildungsausgaben ist als Parteiprogramme. Er findet mit einem quantitativen Längsschnittvergleich von OECD-Daten deutliche Differenzen zwischen tatsächlichen Bildungsausgaben und Parteipositionen in Parteiprogrammen. Wird die Hochschulbildung allein betrachtet, beobachten $\mathrm{Bu}-$ semeyer et al. (2013), dass Parteien des rechten Spektrums eher Befürworter eines Ausbaus der Hochschulfinanzierung sind, was die Autoren damit begründen, dass Hochschulen überproportional von Wohlhabenden aufgesucht werden. Dies wird auch von der Beobachtung untermauert, dass in Schweden ab dem Zeitpunkt des mehrheitlichen Besuchs einer Hochschule durch die Bevölkerung der Ausbau des Hochschulsystems zum Flaggschiff der linken Parteien wurde (Ansell 2008). $\mathrm{Zu}$ abweichenden Ergebnissen bezüglich der Hochschulfinanzierung kommt jedoch Busemeyer (2009a) anhand einer Zeitreihenanalyse von OECD-Staaten, die zeigt, dass Sozialdemokraten die Bildungsausgaben insbesondere für die Hochschulbildung erhöht haben. Dies interpretiert der Autor dahingehend, dass Parteien nicht einfach Transmissionsinstrument für die ökonomischen Interessen sozialer Klassen sind, sondern Politiken und Ausgaben nutzen, um (neue) Wählergruppen anzusprechen und zusammenzuführen. Eine innovative Herangehensweise zur Untersuchung von Bildungsausgaben wählen Garritzmann und Seng (2016), indem sie in ihrer qualitativen Untersuchung von Regierungsprogrammen ganze Regierungsperioden (und nicht einzelne Jahre) betrachten und auf die Zuordnung einzelner Parteien zu Parteienfamilien verzichten. Ihre Ergebnisse können keine Parteieneffekte (mehr) bezüglich der Bildungsausgaben feststellen. Dies wird damit erklärt, dass heutige Bildungssysteme in den 1950er und 1980er-Jahren geprägt wurden und über die Zeit eine Pfadabhängigkeit entwickelten, die radikalen politischen Wandel unwahrscheinlich macht (Busemeyer 2014).

13 Festzustellen ist auch, dass für den untersuchten Zeitraum keine Parteien die Senkung von Bildungsausgaben fordern (Jakobi 2011). 


\section{Exkurs: Multikausale Analysen zur Bildungsausgaben}

Veröffentlichungen zur Höhe von Bildungsausgaben arbeiten meist mit OECD-Datensätzen und multikausalen Modellen, in die gleich mehrere Erklärungsansätze einfließen - nicht allein die Parteiendifferenztheorie. Schmidt (2007) kann zeigen, dass Bildungspolitik als Politikbereich zwischen 1960 und 2002 mehrheitlich Kürzungen unterworfen war, jedoch nicht in allen untersuchten Staaten. Kürzung, Erhalt und Erhöhung von Bildungsausgaben hingen u. a. von gegenseitigen Anpassungsprozessen zwischen Staaten, institutionellen Prozessen, politischen Prozessen in eher staatlich oder eher marktorientierten Staaten, der Zugehörigkeit zu englischsprachigen Staaten und von der parteipolitischen Zusammensetzung von Regierungen ab. Als Determinanten für öffentliche Bildungsausgaben von OECD-Staaten zwischen 1980 und 2001 konnte Busemeyer (2007) als die wichtigsten erklärenden Variablen die wirtschaftliche Entwicklung, den Anteil junger Menschen an der Bevölkerung, die geringe Zahl von Veto-Spielern, die Höhe von öffentlichen Sozialausgaben und die Fiskaldezentralisierung feststellen (Nikolai 2007). Bei einer getrennten Analyse nach Jahrzehnten stellt er fest, dass die Beteiligung konservativer Parteien an der Regierung lediglich in den 1980er-Jahren einen Einfluss auf Bildungsausgaben hatte. Bei Arbeiten, die sich mit der Aufteilung der Bildungsausgaben in öffentlicher und privater Verantwortlichkeit beschäftigen, zeigen Wolf (2009) und Wolf und Zohlnhöfer (2009) für 28 bzw. 26 OECD Staaten im Jahr 2002, dass konservative und christliche Mitte-Parteien als Regierungsparteien, ausgeprägter Protestantismus, Föderalismus und Fiskaldezentralisierung, hohe öffentliche Subventionen für private Bildungszwecke, Staatsverschuldung sowie betriebliche Berufsbildungssysteme negativen Einfluss auf öffentliche Bildungsausgaben haben. Einen positiven Effekt auf die Höhe privater Bildungsausgaben zeigen Föderalismus, Gewerkschaften, liberale Parteien ${ }^{14}$ und Fiskaldezentralisierung, während u. a. christdemokratischen Regierungsparteien, Korporatismus sowie stark ausgeprägter Katholizismus und die Existenz von Kirchensteuern negative Effekte haben.

Weitere Beiträge, die mit der Parteiendifferenztheorie arbeiten, fokussieren die Privatisierung von Bildung und die Integration neoliberaler Elemente in die Bildungspolitik. Mit der Privatisierung von Bildung und Erklärungsmöglichkeiten durch Parteiendifferenzen beschäftigt sich Wiborg (2015) im Rahmen von Schulbildung in England und Schweden. Die qualitative Analyse von Parteiprogrammen zeigt, dass Parteipositionen zur Privatisierung vom Parteiensystem (England: Zweiparteiensystem, Schweden: Mehrparteiensystem) beeinflusst werden. Dies führte dazu, dass es in Schweden zu einem größeren Ausbau von Privatschulen als in England kam. Auch Zehavi (2012) beschäftigt sich mit der Privatisierung der ursprünglich öffentlichen Leistungen im Bildungsbereich und Parteieneffekten. Die Ergebnisse von Experteninterviews und Dokumentenanalysen zeigen, dass Parteien des rechten Flügels die Privatisierung eher vorantreiben als linke Parteien und diese in die politische Agenda aufnehmen. Die Einführung neoliberaler Elemente in die nationale Bildungspolitik und der Effekt von Parteien wird unter anderem von Arnesen und Lundahl (2006)

14 Jensen (2011) zeigt hingegen, dass die Steigerung von Bildungsausgaben nicht mit der Beteiligung linker Parteien in der Regierung verknüpft ist, sondern mit Deindustrialisierung. 
für inklusive Bildung mit quantitativen Analyse nationaler Statistiken in skandinavischen Ländern untersucht. Sie kommen zu dem Schluss, dass die Einführung von neoliberalen Elementen von Regierungsparteien jeglicher Couleur nicht aufgehalten wurde, was sie mit geringen Parteiendifferenzen durch eine wachsende Mittelschicht erklären. Für die schulische Bildung stellt Wiborg (2013) in einer qualitativen Untersuchung hingegen fest, dass Variation zwischen Staaten bezüglich der Einführung neoliberaler Elemente durch regierende Parteien z. T. erklärt werden können.

Insgesamt beschäftigt sich der überwiegende Anteil der Beiträge mit Bildungsausgaben und damit dem parteipolitischen Einfluss auf den bildungspolitischen Output. Dadurch, dass mit den Daten des Manifesto-Projekts auch ein großer qualitativer international-vergleichender Datensatz vorhanden ist, arbeiten die Beiträge mit quantitativen und qualitativen Ansätzen, die sowohl die Bedeutung von Bildungsausgaben in Parteiprogrammen als auch deren Höhe in Zahlen analysieren können.

\subsection{Globalisierung und Europäisierung}

Unter Globalisierung wird die zunehmende Verflechtung zwischen Staaten in unterschiedlichen Bereichen verstanden, die auf wirtschaftliche Liberalisierung des Handels und technische Innovation zurückgeht. Trotz gewisser Ähnlichkeiten ist die Europäisierung von der Globalisierung deutlich abzugrenzen, da die Europäisierung die Wechselwirkungen zwischen ,nationalstaatlicher und supranationaler Ebene des Mehrebenensystems der Europäischen Union“ kennzeichnet (Börzel und Panke 2015, S. 225). Bei der Europäisierung wird in den ausgewählten Veröffentlichungen thematisiert, wie sich die europäische Ebene auf Staaten auswirkt, wobei differenziert werden muss zwischen direkter („harter“) Europäisierung durch Gesetze und rechtlich verbindlichen Entscheidungen sowie ,weichen“ Formen der Europäisierung, wie der Offenen Methode der Koordinierung (OMC). Eine für viele Autoren entscheidende Frage ist die nach Konvergenz (Knill 2005 ${ }^{15}$ ) bzw. Divergenz zwischen den Mitgliedsstaaten bezüglich der nationalen Bildungspolitik verschiedener Bildungsbereiche, da eine Harmonisierung bzw. Angleichung der nationalen Politiken Ziel oder zumindest mögliche Wirkung von Europäisierung ist (ebd., S. 233). Die Beiträge arbeiten dabei vornehmlich mit qualitativen Methoden (insbesondere Dokumentenanalysen, vereinzelt Experteninterviews) mit kleinen Länderfallzahlen und fokussieren den bildungspolitischen Input und Entscheidungsprozess.

Jakobi und Teltemann (2011) stellen mit ihrer quantitativen Analyse verschiedener Datensätze zu OECD-Staaten von 1999 bis 2008 fest, dass die untersuchten Länder in den Bildungsausgaben Konvergenzen zeigen (insbesondere in der Hochschulbildung), wobei es auch Länder gibt, die von diesen Trends abweichen. ${ }^{16}$ Christ

\footnotetext{
15 Hiernach tritt Konvergenz zwischen Staaten u. a. deshalb auf, da zur Lösung eines Problems auf Lösungsstrategien anderer Länder zurückgegriffen wird, internationale Regulationen auferlegt werden, Harmonisierungsprozesse (z. B. EU) auftreten, internationaler Wettbewerb zur Angleichung der Systeme führt (z. B. durch Einigung auf gemeinsame Regeln) oder verstärkter Austausch (Best Practice) stattfindet.

16 In einer früheren Arbeit zeigte Schmidt (2007), dass sich Entwicklungen der Bildungsausgaben nicht mit Globalisierungsprozessen erklären lassen, da Bildungspolitik als Teil der Innenpolitik eines Landes weitgehend von landesspezifischen Demokratisierungsprozessen, Parteipolitik und der institutionellen Ausgestaltung geprägt ist.
} 
und Dobbins (2015) können in ihrer quantitativen Analyse verschiedener Datensätze Internationalisierung als mögliche Ursache von Dezentralisierung der Sekundarbildung in westlichen OECD-Staaten ausschließen.

Andere Beiträge fokussieren den Einfluss der inter- und supranationalen Ebene auf die nationalen Akteure und bildungspolitischen Prozesse. Mit dem Einfluss von Steuerungsinstrumenten inter- und supranationaler Organisationen (z. B. OMK, Beratungsleitungen, international-vergleichende Statistiken, Benchmarks), wie OECD und EU, beschäftigen sich Jakobi et al. (2010a) mit einer quantitativen Analyse verschiedener Datensätze für 30 OECD-Staaten. Sie zeigen, dass im Bereich der formalen Bildung Steuerungsinstrumente Einfluss auf nationale Bildungspolitiken nehmen, deren Wirkung abhängig von den spezifischen Transformationskapazitäten eines Landes ist. $\mathrm{Zu}$ einem ähnlichen Ergebnis für den Bildungsbereich allgemein kommen anhand eines qualitativen Vorgehens Wilkoszewski und Sundby (2016) zu harten und weichen Methoden der Steuerung durch die EU. Sie zeigen, dass deren Erfolg entscheidend von den involvierten Stakeholdern abhängt. Trampusch (2009) greift thematisch das Einfließen europäischer Politik zur beruflichen Aus- und Weiterbildung in nationale Bildungspolitik in Deutschland und Österreich auf. Mit einer qualitativen Analyse zeigt sie, dass Europäisierung unterschiedlich erfolgt: durch Reformpolitiken (DE) oder als Nebenprodukt des nationalen institutionellen Wandels (AT). Den Einfluss internationaler Organisationen auf nationale bildungspolitische Netzwerke untersucht Nagel (2010) für Deutschland, die Schweiz, England und Neuseeland mit einem Methodenmix. Er stellt Wandlungsprozesse bezüglich der Ziele, Instrumente und des Rahmens von Bildungspolitik sowie Änderungen institutioneller Strukturen fest, während der politische Entscheidungsprozess von Bildung über die Zeit sehr konstant geblieben ist. Die bildungspolitischen Akteure eines Landes fördern politische Initiativen der internationalen Ebene im eigenen Interesse, zeigen sich gleichzeitig jedoch sehr konservativ, wenn es um die Zusammensetzung des eigenen politischen Netzwerks geht oder die politischen Interaktionsmodi.

Mit dem Einfluss der inter- und supranationalen Ebene auf die Inhalte nationaler Bildungspolitik (Output) beschäftigt sich beispielsweise Ioannidou (2010), die den steuernden Einfluss von EU und der OECD auf das Konzept des LLL in Deutschland, Finnland und Griechenland untersucht. Sie macht mit ihrer qualitativen Analyse von Experteninterviews und Dokumenten deutlich, dass die Organisationen mit unterschiedlichen Handlungsressourcen (EU: Finanzierung, rechtliche Regelungen, Monitoring; OECD: Empfehlungen, Evaluationen, Studien und Bildungsberichterstattung) steuernd Einfluss auf nationale Politiken zu LLL nehmen. Cavaco et al. (2014) untersuchen die Entwicklung von Anerkennungsinstrumenten für die informelle und non-formale Erwachsenenbildung in Portugal und Frankreich vor dem Hintergrund des Einflusses der EU-Bildungsagenda mit Experteninterviews. Sie stellen fest, dass die EU das Thema, welches ursprünglich von der Zivilgesellschaft ausging, inkorporierte und mit einer wirtschaftlichen Orientierung versah. Sie können zeigen, dass die Anerkennungspolitik in beiden Ländern nicht von der EU dominiert wird, sondern aus dem Zusammenspiel transnationaler und nationaler Einflüsse resultiert. Nationale Einflüsse beim Aufgreifen europäischer Bildungspolitik stellen auch andere Autoren mit qualitativen Untersuchungen für die Hochschulpolitik (Dobbins und Knill 2009) und Studiengebühren an Hochschulen (Förster 
2011) fest und führen diese auf das jeweilige politische Erbe und unterschiedliche wohlfahrtsstaatliche Traditionen sowie Unterschiede zwischen Parteien innerhalb der Staaten zurück. Demgegenüber stellen Capano und Piattoni (2011) mit ihrer ebenfalls qualitativen Analyse fest, dass insbesondere die Lissabon-Strategie in England, den Niederlanden, Deutschland und Italien ein Konzept lieferte, welches nationale, kulturelle und politische Traditionen und Vorstellungen zur Hochschulbildung und -politik zu überwinden half.

Zusammenfassend greifen die beschriebenen Beiträge insbesondere das Thema der neuen Bildungssteuerung auf sowie den Einfluss inter- und supranationaler Akteure auf nationale Akteure und die Ausgestaltung nationaler Bildungspolitiken. Während es für quantitative Studien schwierig ist, Internationalisierung als Einflussfaktor zu operationalisieren, können qualitative Arbeiten diese Entwicklungen mit Dokumentenanalysen und Experteninterviews gut nachzeichnen. Jedoch führen die kleinen Länderfallzahlen und die kleine Anzahl von Publikationen zu ähnlichen Fragestellungen dazu, dass verallgemeinerbare Tendenzen, beispielsweise für den europäischen Bildungsraum, schwer abzuleiten sind.

\subsection{Pfadabhängigkeit}

Die Grundannahme hinter dem Ansatz der Pfadabhängigkeit ist, dass zurückliegende Ereignisse den Ablauf von Prozessen beeinflussen oder sogar determinieren können. Beyer (2015, S. 149f.) führt an, dass unterschiedliche Aspekte als Phänomene der Pfadabhängigkeit gesehen werden, wie z. B. die große Relevanz früherer Ereignisse, die Bedeutung von geringfügigen Ereignissen und Zufällen, die Zwangläufigkeit von einmal eingeschlagenen Entwicklungen durch positive Rückkopplungen, die begrenzte Rationalität von Akteuren sowie hohe Transaktionskosten bei Veränderungen. North (1990) nutzt die Pfadabhängigkeit als einen Bestandteil seiner Theorie zum institutionellen Wandel. Das Konzept wird in der ausgewählten Literatur z. T. als Ergänzung anderer Ansätze gewählt, wie z. B. der Parteiendifferenztheorie, der Globalisierung und Europäisierung oder dem Akteurzentrierten Institutionalismus. Die überwiegende Zahl der Beiträge arbeitet mit qualitativen Methoden und stellt die Entstehung von Bildungsoutputs durch die Betrachtung des Inputs bzw. politischen Entscheidungsprozesses in den Vordergrund.

Politikerbe als historisch angelegter „Problemlösungspfad“ oder Resultat von Pfadabhängigkeit wird von einigen Autoren als Filter gesehen zwischen Globalisierungs- und Europäisierungseinflüssen und dem politischen Output der Schul- und Hochschulpolitik (Jakobi und Teltemann 2011). Gornitzka und Maassens (2011) zeigen mit ihrer qualitativen Analyse, dass die skandinavischen Länder mit ihrer starken Bildungstradition wenige Bezüge zu europäischen Bildungsagenden aufweisen und entgegen internationaler Trends keine Studiengebühren einführten. Dobbins und Knill (2009) arbeiten mit Experteninterviews heraus, dass europäisch induzierter Isomorphismus durch die starken nationalen Unterschiede in Zentral- und Osteuropa zu unterschiedlichen nationalen Ergebnissen in der Ausgestaltung von Hochschulpolitik führt. Mit einer qualitativen Untersuchung zu britischen und deutschen Universitäten und den Auswirkungen von Internationalisierung zeigt Graf (2009), dass es in Deutschland statt zu einer Pfadverfestigung eher zu einer Abweichung und 
zur internationalen Öffnung kommt, um das Hochschulsystem anschlussfähiger zu machen, während in Großbritannien eine Pfadstabilisation der Internationalisierung der Hochschule einsetzt, da diese schon eine lange Tradition hat. Bezogen auf das von supra- und internationalen Organisationen (EU, OECD) beworbene LLL stellt Ioannidou (2010) mit Experteninterviews und Dokumentenanalysen für Deutschland, Griechenland und Finnland ebenfalls eine Filterung dieses Konzepts durch nationale kulturell und historisch geprägte Bildungstraditionen fest. Mit einer qualitativen Rekonstruktion von Schulcurricula in England und Frankreich in Bezug auf den Einfluss europäischer Themen zeigt Haus (2009), dass sich in Frankreich ein unterstützender Pfad in Form institutioneller Strukturen zeigt, der ein Einfließen europäischer Inhalte in nationale Politiken zulässt, während dies in England nicht der Fall ist.

Unabhängig von Internationalisierungstendenzen dient der Ansatz auch zur Erklärung von Stabilität nationaler Bildungspolitik (Outputs). So untersucht Hillmert (2008) die unterschiedliche Entwicklung der Berufsbildungssysteme in England und Deutschland anhand der Auswertung nationaler Bildungsberichte und bescheinigt beiden Systemen eine große Stabilität, die Wandel nur bei triefgreifenden wirtschaftlichen Veränderungen zulässt. Baldi (2012) untersucht in diesen Ländern ebenfalls qualitativ unterschiedliche Praxen zur Nicht-Aufteilung (E) und Aufteilung (D) der Schülerschaft in der Sekundarstufe. Er stellt fest, dass in England das Einfließen neuer Bildungsideen zu Reformen führte, während in Deutschland verfestigte institutionelle politische Strukturen (begleitet vom Föderalismus in der Bildung) Reformen verhinderte. Nikolai (2007) zeigt mit ihrer quantitativen Längsschnittanalyse von OECD-Staaten, dass die Bildungsausgaben eines Landes stark von vergangenen Bildungsausgaben abhängen. Pfadabhängigkeit bzw. Politkerbe wird bei Analysen der Manifesto-Daten zu Parteiprogrammen auch als Erklärung dafür gesehen, welche Parteien in Staaten einen Ausbau der Bildungsausgaben befürworten (Busemeyer et al. 2013) und dass Parteieneffekte auf die Bildungsausgaben abnehmen (Garritzmann und Seng 2016).

Was das Verlassen des politischen Pfades und institutionellen Wandel begünstigen kann, untersucht Trampusch (2009) zum einen in Bezug auf den Einfluss europäischer Politik zur beruflichen Aus- und Weiterbildung auf nationale Reformen in Deutschland und Österreich und, zum anderen, in Bezug auf die Entwicklung der beruflichen Aus- und Weiterbildung in Deutschland, Österreich und der Schweiz (Trampusch 2010a). Sie zeigt, dass Arbeitgeberkonstellationen bzw. Firmeninteressen und die Führungsebene der öffentlichen Bildungsverwaltung die Art des institutionellen Wandels beeinflussen. Sind große Unternehmen die dominierenden Akteure, die mit der öffentlichen Bildungsverwaltung kooperieren, ist ein umformender institutioneller Wandel zu beobachten. Wenn KMU die dominierenden Akteure stellen, die mit den öffentlichen Bildungseliten in Interaktion treten, sind selbsterhaltende politische Prozesse festzustellen. Weitergehend zeigen die Ergebnisse, dass insbesondere KMU und nicht Gewerkschaften als Bremse für einen umformenden Wandel agieren.

Zusammenfassend nutzen Beiträge Pfadabhängigkeit zu einem als Erklärung für die Stabilität nationaler Bildungspolitik (auch vor dem Hintergrund internationaler 
Einflüsse) und zum anderen beschäftigen sich einige der Beiträge mit der Frage, wann ein politischer Pfad verlassen wird und es zum institutionellen Wandel kommt.

\subsection{Akteurzentrierter Institutionalismus (AZI)}

Der AZI wurde von Mayntz und Scharpf Mitte der 1990er-Jahre entwickelt. Er kann als „Forschungsheuristik“ oder multikausal angelegter Analyserahmen gesehen werden, der Ablauf und Ergebnis von politischen Entscheidungsprozessen mithilfe der beteiligten Akteure, ihrer Konstellation und des institutionellen Kontextes (Scharpf 2006, S. 76 f.) erklärt. Der Ansatz differenziert zwischen Akteurstypen, Interaktionsformen (einseitiges Handeln, Verhandlung, Mehrheitsentscheidung, hierarchische Steuerung, Verhandeln im Schatten der Hierarchie) und Handlungsressourcen von Akteuren (ebd., S. $86 \mathrm{ff} ., 101 \mathrm{ff}$.). In der ausgewählten Literatur beschäftigen sich die Autoren mit dem politischen Input bzw. Willensbildungs- und Entscheidungsprozess. Sie identifizieren Ursachen, die zum Auftreten unterschiedlicher nationaler Systeme führen, und nutzen dabei qualitative Forschungsansätze wie beispielsweise Experteninterviews und Dokumentenanalysen (Knauber und Ioannidou 2016; Ioannidou 2010; Witte et al. 2008).

Knauber und Ioannidou (2016) fokussieren nationale Akteure der Grundbildungspolitik in England, Österreich, den Niederlanden und der Türkei und zeigen, dass die Interaktionsformen der Akteure von der jeweiligen Steuerung von Grundbildungspolitik abhängen: Bei zentralistischer Governance werden Entscheidungen begünstigt, die durch hierarchische Steuerung herbeigeführt werden und weniger durch Verhandlungen oder Mehrheitsentscheidungen, wie in Staaten mit regionalen und lokalen Steuerungsmodellen. In einigen Staaten können nicht-staatliche Akteure durch spezifische Handlungsressourcen begrenzten Einfluss auf den politischen Entscheidungsprozess und die Implementation nehmen. Die Auswirkungen des BolognaProzesses auf höhere universitäre und nicht-universitäre Bildung in Deutschland, Frankreich und den Niederlanden mit einem Fokus auf nationale Akteure betrachten Witte et al. (2008). Sie arbeiten heraus, dass insbesondere Akteure der Ministerien nationale Reformprozesse beschleunigt haben. Ioannidou (2010) nutzt den AZI zur Untersuchung des steuernden Einflusses der OECD und EU auf LLL in Deutschland, Finnland und Griechenland. Sie stellt fest, dass sich Steuerungsbemühungen zum LLL durch spezifische Handlungsressourcen (wie Bildungsberichterstattung und Monitoring) durch verschiedene Akteure unterschiedlicher Ebenen (regional, national, transnational) beobachten lassen. Die identifizierten Schlüsselakteure treten immer in Konstellationen mit wechselseitigen Interdependenzen auf und zeigen Interaktionen auf Grundlage von Verhandlungen und Mehrheitsregelungen. Afdahl (2013) untersucht den politischen Prozess der Lehrerbildung in Finnland und Norwegen mit einem Fokus auf involvierte Akteure und zeigt, dass Lehrerbildung in Norwegen stark von den beteiligten politischen Akteuren und Bedingungen abhängt, während finnische Politik zur Lehrerbildung eher von akademischer Expertise bei der Ausformulierung von Politik beeinflusst wird. Eine qualitative Untersuchung der Auswirkung von Reformen im Föderalismus auf die Hochschulpolitik in Deutschland und der Schweiz stellen Griessen und Braun (2010) dar. Sie zeigen, dass generelle Reformen in Deutschland wenig Einfluss auf föderale Koordinationsstruk- 
turen in der Hochschulpolitik hatten, während in der Schweiz mehr Verflechtungen zu finden sind, gleichzeitig aber institutionelle Mechanismen, die politischen Reformblockaden entgegenwirken. In beiden Ländern sind unilaterale Strategien von Akteuren kaum möglich, wohingegen kollektive Vereinbarungen föderaler Akteure erfolgversprechender sind.

Zusammenfassend thematisieren Beiträge zum AZI in erster Linie Fragen zur Steuerung von Bildungspolitik und Akteuren und wie diese zu bildungspolitischen Outputs beitragen.

\section{Fazit zu der ausgewählten Literatur und ihrer thematischen und theoretischen Fokussierung}

Die Mehrheit der in den Beiträgen analysierten qualitativen Daten wurde selbst erhoben (Interviews) bzw. recherchiert (politische Dokumente); einige Arbeiten stützen sich auf Daten, die im Rahmen des Manifesto-Projekts gesammelt werden, welches Parteiprogramme inhaltsanalytisch auswertet. Die Mehrheit der quantitativen Arbeiten stützt sich auf die Analyse bereits vorhandener Datensätze, vorwiegend von der OECD (Education at a Glance-Datenbank, PISA, IALS/PIAAC), der Weltbank, der UNESCO, Eurostat (AES, Education and Training Database, EU-LFS) und vom Eurydice-Netzwerk. Das thematische Spektrum der Beiträge ist weit gefächert. Ein deutlicher Schwerpunkt bei den Bildungsbereichen ist die formale Bildung mit Beiträgen zu Schule (12\%), Hochschule (20\%), beruflicher Aus- und Weiterbildung $(22 \%)$ oder übergreifenden Beiträgen zur formalen Bildung (6\%). Demgegenüber beschäftigen sich lediglich $12 \%$ der Beiträge mit Erwachsenen- und Weiterbildung bzw. LLL. Die restlichen Beiträge sind keinem der Bildungsbereiche direkt zuzuordnen, da sie sich beispielsweise bereichsübergreifend mit Bildungsausgaben beschäftigen. Nach Themen sortiert beschäftigen sich $35 \%$ der Beiträge mit bildungspolitischem Input (z. B. institutionelle Ausgestaltung von Bildungspolitik), $37 \%$ mit Output (z. B. Bildungsausgaben) und $18 \%$ der Beiträge mit Outcome (z. B. Bildungsteilnahme, Bildungsleitung, Bildungschancen, Stratifizierung). Die restlichen Beiträge sind nicht eindeutig zuzuordnen. Bei den Thematiken der Bildungspolitik, die von den verschiedenen Theorien vorwiegend adressiert werden, lassen sich zwei Lager herausarbeiten: Für Beiträge, die sich mit Bildungsoutput und -outcome beschäftigen, bewähren sich die Wohlfahrtsstaatentheorie, VoC und die Parteiendifferenztheorie; für Beiträge, die sich überwiegend mit dem Input bzw. dem politischen Willensbildungs- und Entscheidungsprozess befassen, bewähren sich Pfadabhängigkeit, Globalisierung und Europäisierung und Akteurzentrierter Institutionalismus (vgl. Tab. 1). Methodisch lassen sich diese beiden Stränge ebenfalls gut unterscheiden. Output- und Outcome-orientierte Beiträge forschen eher mit quantitativen Methoden mit Ausnahme der Parteiendifferenztheorie. Input-orientierte Veröffentlichungen wählen mehrheitlich ein qualitatives Forschungsdesign. Beiträge zum Bildungsbereich Erwachsenen- und Weiterbildung bzw. LLL arbeiten sowohl mit qualitativen als auch mit quantitativen Forschungsdesigns und nehmen primär Input und Outcome in den Blick, weniger den Output von Bildungspolitik. 
Tab. 1 Anzahl der Beiträge nach Theorien, Bildungsbereichen und Themen (Input, Output, Outcome), Mehrfachnennung möglich

\begin{tabular}{|c|c|c|c|c|c|c|}
\hline & $\begin{array}{l}\text { Wohlfahrts- } \\
\text { staaten }\end{array}$ & VoC & $\begin{array}{l}\text { Parteien- } \\
\text { differenz- } \\
\text { theorie }\end{array}$ & $\begin{array}{l}\text { Globalisierung } \\
\text { \& Europäi- } \\
\text { sierung }\end{array}$ & $\begin{array}{l}\text { Pfadabhän- } \\
\text { gigkeit }\end{array}$ & AZI \\
\hline $\begin{array}{l}\text { Sekundär- } \\
\text { bildung }\end{array}$ & 2 & 1 & 3 & 1 & 2 & 1 \\
\hline $\begin{array}{l}\text { Berufliche } \\
\text { Ausbildung }\end{array}$ & 1 & 9 & - & 1 & 3 & - \\
\hline $\begin{array}{l}\text { Universitäre } \\
\text { Ausbildung }\end{array}$ & 3 & 1 & 2 & 3 & 3 & 2 \\
\hline $\begin{array}{l}\text { Erwachsenen- } \\
\text { bildung, } \\
\text { LLL, Wei- } \\
\text { terbildung }\end{array}$ & 3 & 2 & - & 2 & 1 & 2 \\
\hline Übergreifend & 6 & 2 & 11 & 4 & 4 & - \\
\hline $\begin{array}{l}\text { Input \& } \\
\text { Prozess }\end{array}$ & - & 3 & 1 & 9 & 10 & 4 \\
\hline Output & 6 & 3 & 15 & 2 & 3 & - \\
\hline Outcome & 8 & 4 & - & - & - & - \\
\hline
\end{tabular}

\section{Diskussion}

Diese Literatur-Review zielt darauf, Beiträge der vergangenen zehn Jahre systematisch zu analysieren, die sich mit bildungspolitischen Themen international-vergleichend beschäftigen und dabei Ansätze und Theorien der vergleichenden Politikwissenschaft verwenden. Zu den in den Veröffentlichungen identifizierten Ansätzen gehören die Wohlfahrtsstaatentheorie, Varieties of Capitalism, Parteiendifferenztheorie, Globalisierung und Europäisierung, Pfadabhängigkeit und der Akteurzentrierte Institutionalismus - die meisten davon Ansätze, die dem Bereich der Policy-Forschung zuzuordnen sind. Die Ansätze werden von den Autoren zum Teil für einen Bildungsbereich modifiziert. Auch werden die Ansätze in den ausgewählten Veröffentlichungen nicht immer exklusiv verwendet, sondern miteinander verwoben und kombiniert.

Einschränkungen der Beiträge ergeben sich auf unterschiedlichen Ebenen, die die Untersuchungsgegenstände und auch die Daten betreffen. Die überwiegende Zahl der Beiträge konzentriert sich nur auf einen Bildungsbereich des Politikfelds Bildungspolitik. Es ist jedoch einerseits davon auszugehen, dass es höchstwahrscheinlich Interaktionen zwischen Bildungsbereichen und andererseits auch zwischen Bildungspolitik und anderen Politikfeldern gibt. Eine weitere Einschränkung einiger quantitativ arbeitender Veröffentlichungen ist, dass nicht auf Zeitreihen zurückgegriffen wird, sondern Daten eines Erhebungszeitpunktes verwendet werden. Dies suggeriert die Stabilität von Bildungssystemen über die Zeit. Wird ein Blick auf die verwendeten Datensätze geworfen, so fällt auf, dass die Anzahl und die Qualität vorhandener internationaler Datensätze zu Bildungsthemen in den vergangenen Jahren stetig zunahmen. Zu berücksichtigen ist dabei, dass viele der verwendeten Datensätze aus den gleichen Bezugsquellen stammen und insbesondere die OECD 
eine Art „Datenmonopol“ aufgebaut hat. Im multidisziplinären Forschungsfeld der Bildungspolitik ist die Gewichtung der Forschungsthemen deutlich abhängig von dem Bestand und der Aussagekraft von Datensätzen. Bisher überwiegen Datensätze zu formaler Bildung, deren (öffentlicher und privater) Finanzierung und deren Teilnahmezahlen sowie zur Bildungsleistung in formaler Bildung. Für die Erwachsenen- und Weiterbildung liegen bisher nur wenige internationale Datensätze vor, was sich auch in der geringen Anzahl von Veröffentlichungen widerspiegelt. Für die Erwachsenen- und Weiterbildung, die meist im non-formalen und informellen Bereich stattfindet, fehlen für den internationalen Vergleich aussagekräftige Datensätze zu spezifischeren Themen und Variablen, wie Anbieter, Angebote, Personal, Qualität oder Finanzierung. Erst darauf aufbauend können ähnlich belastbare Berechnungen wie die beschriebenen zur formalen Bildung durchgeführt sowie Typologien von Staaten entwickelt werden. International-vergleichende Daten zu Kompetenzen Erwachsener gibt es zudem erst seit IALS, ALL und PIAAC. Allerdings wurden diese bisher kaum in den ausgewählten Veröffentlichungen verwendet, während deren Verwendung in anderen Forschungsfeldern bereits verbreitet ist. ${ }^{17}$ Dies könnte zum einen daran liegen, dass (bisher) noch keine internationalen Längsschnittdaten vorliegen die Kausalanalysen ermöglichen. Da es im Erwachsenenalter keine zentrale Bildungsinstitution gibt, können zum anderen sowohl die Gründe für bestimmte Kompetenzausprägungen als auch die Effekte von Kompetenzausprägungen sehr divers sein, wodurch vergleichende Modelle äußerst komplex werden. Die berufliche Bildung, die Überschneidungen zur Erwachsenen- und Weiterbildung aufweist, bildet hier, was Daten und Veröffentlichungen angeht, eine positive Ausnahme.

Die aufgezeigten theoretischen Ansätze bieten gleichwohl Anknüpfungsmöglichkeiten für die international-vergleichende Erwachsenenbildungsforschung und die Erforschung von Bildungspolitik, haben für diese jedoch auch unterschiedliche Erträge. Wie bereits im Fazit dargestellt, unterscheiden sich die beschriebenen Ansätze und Theorien und die mit ihnen beforschten Themen. Wohlfahrtsstaatentheorie, VoC und Parteiendifferenztheorie nehmen in erster Linie den bildungspolitischen Output und Outcome in den Blick (und welche Variablen diesen beeinflussen) und arbeiten primär mit quantitativen Methoden und großen Fallzahlen. Für die international-vergleichende Erwachsenenbildungsforschung kann hier eine Option in der Untersuchung des Zusammenhangs von bildungspolitischem Output und Outcome liegen. So können beispielsweise Erträge oder auch wider benefits von bestimmten (Bildungs-)Politiken festgestellt werden, wie von nationalen und internationalen Politiken zur Förderung spezieller Adressaten (z. B. Grundbildung für Geringqualifizierte), zur Qualitätssicherung oder Zertifizierung. Allerdings muss u. a. bei der Wohlfahrtsstaatentheorie eine argumentative Verbindung zur (Erwachsenen-)Bildung noch geleistet werden, weshalb sich ein Rückgriff auf die theoretischen Modifikationen bzw. Weiterentwicklungen des Ansatzes empfiehlt, die Bildung stärker in den Fokus setzen. Mit ihrer Komplexitätsreduktion erleichtern Typologien des Wohlfahrtsstaaten- und VoC-Ansatzes den Vergleich bei großer Länderfallzahl. In Bezug auf die Erwachsenenbildung mit ihrem Mehrebenensystem (Schrader 2011) kann

17 Siehe dazu auch Veranstaltungen zur Forschung mit PIAAC-Daten: http://www.oecd.org/skills/piaac/ neweventspage.htm (abgerufen am 08.06.2017). 
dies jedoch zu einer verkürzten Darstellung der Besonderheiten eines Staates und zur Ableitung falscher Folgerungen führen, gerade wenn eher der bildungspolitische Input oder Prozess im Fokus der Forschung liegen. ${ }^{18}$

Für den bildungspolitischen Input und Prozess eignen sich hingegen andere Ansätze, wie Globalisierung und Europäisierung, Pfadabhängigkeit und Akteurzentrierter Institutionalismus. Diese fokussieren die Ursachen für die Entstehung spezieller Bildungssysteme und nationaler Bildungspolitiken und arbeiten meist qualitativ mit kleinen Fallzahlen, theoriebildend, um die Spezifika der einzelnen Systeme und Politiken adäquat darstellen zu können. Daher sind diese Ansätze besonders ertragreich für neuere Themen der Erwachsenenbildungsforschung, wie Internationalisierung (Einfluss bzw. Steuerung inter- und supranationaler Akteure), komplexe Interaktionsgeflechte (Mehrebenensystem) und neue Governance-Strukturen, welche auch die Rolle und den Einfluss zivilgesellschaftlicher Akteure für die Bildungspolitik beleuchten. Diese Ansätze sind auch deshalb ergiebig, da sie Bildungssysteme und -politiken wissenschaftlich beschreiben, systemübergreifend vergleichen und dadurch auch zur Weiterentwicklung von Theorie beitragen können.

Besonders erfolgversprechend sind einige jüngere Forschungsarbeiten, die mit Methodenmix-Designs und mehreren theoretischen Zugängen bzw. Annahmen arbeiten. In Forschungsarbeiten können so quantitative Analysen von Datensätzen mit großen Länderfallzahlen durch die intensivere Betrachtung einzelner qualitativer Länderfallstudien ergänzt werden. Forschungsergebnisse können damit im Idealfall sowohl die Ursachen für Bildungspolitiken beschreiben als auch deren Wirkungen erklären.

Open Access Dieser Artikel wird unter der Creative Commons Namensnennung 4.0 International Lizenz (http://creativecommons.org/licenses/by/4.0/deed.de) veröffentlicht, welche die Nutzung, Vervielfältigung, Bearbeitung, Verbreitung und Wiedergabe in jeglichem Medium und Format erlaubt, sofern Sie den/die ursprünglichen Autor(en) und die Quelle ordnungsgemäß nennen, einen Link zur Creative Commons Lizenz beifügen und angeben, ob Änderungen vorgenommen wurden.

\section{Literatur}

Afdal, H.W. (2013). Policy making processes with respect to teacher education in Finland and Norway. Higher Education, 65(2), 167-180.

Ansell, B.W. (2008). University challenges: explaining institutional change in higher education. World Politics, 60(02), 189-230.

Ansell, B. W. (2010). From the ballot to the blackboard: the redistributive political economy of education. New York: Cambridge University Press.

Arnesen, A.-L., \& Lundahl, L. (2006). Still social and democratic? Inclusive education policies in the nordic welfare states. Scandinavian Journal of Educational Research, 50(3), 285-300.

Baldi, G. (2012). Schools with a difference: policy discourses and education reform in Britain and Germany. West European Politics, 35(5), 999-1023.

Beblavý, M., Thum, A.-E., \& Veselkova, M. (2013). Education and social protection policies in OECD countries: social stratification and policy intervention. Journal of European Social Policy, 23(5), 487-503.

\footnotetext{
18 Analysen hängen zudem sehr stark davon ab, welche Länder in ein Sample aufgenommen werden, um ein bestimmtes Regime zu repräsentieren. Teils weisen die Länder innerhalb eines Regimetyps starke Variationen auf. Je weniger Länder eines Typus betrachtet werden, desto schwieriger sind die Ergebnisse im Hinblick auf verallgemeinernde Aussagen einzuschätzen.
} 
Beyer, J. (2015). Pfadabhängigkeit. In G. Wenzelburger \& R. Zohlnhöfer (Hrsg.), Handbuch Policy-Forschung (S. 149-172). Wiesbaden: Springer.

von Beyme, K. (2000). Parteien im Wandel. Wiesbaden: Westdeutscher Verlag.

Boeren, E., \& Holford, J. (2016). Vocationalism varies (a lot): a 12-country multivariate analysis of participation in formal adult learning. Adult education quarterly, 66(2), 120-142.

Börzel, T., \& Panke, D. (2015). Europäisierung. In G. Wenzelburger \& R. Zohlnhöfer (Hrsg.), Handbuch Policy-Forschung (S. 225-246). Wiesbaden: Springer VS.

Bosch, G., \& Charest, J. (2008). Vocational training and the labour market in liberal and coordinated economies. Industrial Relations Journal, 39(5), 428-447.

Busemeyer, M. R., Franzmann, S. T., \& Garritzmann, J. L. (2013). Who owns education? Cleavage structures in the partisan competition over educational expansion. West European Politics, 36(3), 521-546.

Busemeyer, M.R. (2007). Determinants of public education spending in 21 OECD democracies, 1980-2001. Journal of European Public Policy, 14(4), 582-610.

Busemeyer, M.R. (2009). Asset specificity, institutional complementarities and the variety of skill regimes in coordinated market economies. Socio-Economic Review, 7(3), 375-406.

Busemeyer, M. R. (2009a). Social democrats and the new partisan politics of public investment in education. Journal of European Public Policy, 16(1), 107-126.

Busemeyer, M. R. (2013). Education funding and individual preferences for redistribution. European Sociological Review, 29(6), 1122-1133.

Busemeyer, M. R. (2014). Skills and inequality: partisan politics and the political economy of education reforms in Western welfare states. Cambridge: Cambridge University Press.

Busemeyer, M. R. (2015). Bildungspolitik. In G. Wenzelburger \& R. Zohlnhöfer (Hrsg.), Handbuch PolicyForschung (S. 615-640). Wiesbaden: Springer VS.

Busemeyer, M.R., \& Trampusch, C. (2011). Review article: comparative political science and the study of education. British Journal of Political Science, 41(02), 413-443.

Capano, G., \& Piattoni, S. (2011). From Bologna to Lisbon: the political uses of the Lisbon „script“ in European higher education policy. Journal of European Public Policy, 18(4), 584-606.

Cavaco, C., Lafont, P., \& Pariat, M. (2014). Policies of adult education in Portugal and France: the European Agenda of validation of non-formal and informal learning. International Journal of Lifelong Education, 33(3), 343-361.

Christ, C., \& Dobbins, M. (2015). Dezentralisierung der Bildung in den westlichen OECD-Staaten: Eine vergleichende Analyse der Ursachen und Formen. In J. Schrader, J. Schmid, K. Amos \& A. Thiel (Hrsg.), Governance von Bildung im Wandel. Interdisziplinäre Zugänge (S. 145-173). Wiesbaden: Springer VS.

Culpepper, P.D. (2007). Small states and skill specificity: Austria, Switzerland, and interemployer cleavages in coordinated capitalism. Comparative Political Studies, 40(6), 611-637.

Culpepper, P.D., \& Thelen, K. (2008). Institutions and collective actors in the provision of training: historical and cross-national comparisons. In K. U. Mayer \& H. Solga (Hrsg.), Skill formation (S. 21-49). Cambridge: Cambridge University Press.

Desjardins, R. (2013). Considerations of the impact of neoliberalism and alternative regimes on learning and its outcomes: an empirical example based on the level and distribution of adult learning. International Studies in Sociology of Education, 23(3), 182-203.

Dobbins, M., \& Knill, C. (2009). Higher education policies in central and eastern Europe: convergence toward a common model? Governance, 22(3), 397-430.

Ebner, C., \& Nikolai, R. (2010). Duale oder schulische Berufsausbildung? Entwicklungen und Weichenstellungen in Deutschland, Österreich und der Schweiz. Swiss Political Science Review, 16(4), 617-648.

Esping-Andersen, G. (1998). The three worlds of welfare capitalism. Princeton: Princeton University Press.

Estevez-Abe, M., Iversen, T., \& Soskice, D. (2001). Social protection and the formation of skills: a reinterpretation of the welfare state. In P. A. Hall \& D. Soskice (Hrsg.), Varieties of capitalism. The institutional foundations of comparative advantage (S. 145-183). New York: Oxford University Press.

Field, J., Künzel, K., \& Schemmann, M. (2016). International comparative adult education research. In M. Schemmann (Hrsg.), Internationales Jahrbuch der Erwachsenenbildung (S. 109-134). Köln: Böhlau.

Förster, C. (2011). Divergenz in der Konvergenz: Studiengebührenpolitik im Europäischen Hochschulraum - die Beispiele Deutschland, England und Österreich. In K. Amos, J. Schmid, J. Schrader \& A. Thiel (Hrsg.), Welten der Bildung? Vergleichende Analysen von Bildungspolitik und Bildungssystemen (S. 179-202). Baden-Baden: Nomos.

Garritzmann, J.L., \& Seng, K. (2016). Party politics and education spending: challenging some common wisdom. Journal of European Public Policy, 23(4), 510-530. 
Gift, T., \& Wibbels, E. (2014). Reading, writing, and the regrettable status of education research in comparative politics. Annual Review of Political Science, 17, 291-312.

Gornitzka, A., \& Maassen, P. (2011). University governance reforms, global scripts and the „Nordic Model“: accounting for policy change? In K. Amos, J. Schmid, J. Schrader \& A. Thiel (Hrsg.), Welten der Bildung? Vergleichende Analysen von Bildungspolitik und Bildungssystemen (S. 149-178). BadenBaden: Nomos.

Graf, L. (2009). Applying the varieties of capitalism approach to higher education: comparing the internationalisation of German and British universities. European Journal of Education, 44(4), 569-585.

Graf, L. (2013). The hybridization of vocational training and higher education in Austria, Germany, and Switzerland. Opladen: Budrich UniPress.

Graf, L. (2016). Betrieblich-hochschulbasierte Ausbildungsformen in Deutschland und den USA: Eine (Re)Konzeptualisierung. Zeitschrift für Pädagogik, 62(3), 323-339.

Green, A., Preston, J., \& Janmaat, J.G. (2006). Education, equality and social cohesion: a comparative analysis. Basingstoke: Palgrave Macmillan.

Griessen, T., \& Braun, D. (2010). Hochschulföderalismus zwischen Kooperationszwang und Blockadegefahr: Deutschland und die Schweiz im Vergleich. Schweizerische Zeitschrift für Politikwissenschaft, 16(4), 715-746.

Hall, P., \& Soskice, D. (Hrsg.). (2001). Varieties of capitalism. The institutional foundations of comparative advantage. New York: Oxford University Press.

Haus, L. (2009). Europeanization, education, and school curricula: the role of historical legacies in explaining policy variation between England and France. Comparative Political Studies, 42(7), 916-944.

Hega, G. M. (2011). Wohlfahrtsstaaten und Bildungspolitik: Nachzügler, Vorreiter oder Ausreißer? Die Schweiz und die Vereinigten Staaten in vergleichender Perspektive. In K. Amos, J. Schmid, J. Schrader \& A. Thiel (Hrsg.), Welten der Bildung? Vergleichende Analysen von Bildungspolitik und Bildungssystemen (S. 53-100). Baden-Baden: Nomos.

Heidenheimer, A.J. (1981). Education and social security entitlements in Europe and America. In P. Flora \& A.J. Heidenheimer (Hrsg.), The development of welfare states in Europe and America (S. 269-304). New Brunswick: Transaction.

Hillmert, S. (2008). When traditions change and virtues become obstacles. Skill formation in Britain and Germany. In K. U. Mayer \& H. Solga (Hrsg.), Skill Formation (S. 50-81). Cambridge: Cambridge University Press.

Hoelscher, M. (2012). Spielarten des Kapitalismus und Kompetenzen von Hochschulabsolventinnen und -absolventen. Kölner Zeitschrift für Soziologie und Sozialpsychologie, 64, 479-505.

Höpner, M. (2015). Spielarten des Kapitalismus. In G. Wenzelburger \& R. Zohlnhöfer (Hrsg.), Handbuch Policy-Forschung (S. 173-198). Wiesbaden: Springer VS.

Hurrelmann, K., Quenzel, G., \& Rathmann, K. (2011). Bildungspolitik als Bestandteil moderner Wohlfahrtspolitik: Deutschland im internationalen Vergleich. Zeitschrift für Soziologie der Erziehung und Sozialisation, 31(3), 313-326.

Ioannidou, A. (2010). Steuerung im transnationalen Bildungsraum: Internationales Bildungsmonitoring zum Lebenslangen Lernen. Bielefeld: wbv.

Iversen, T., \& Stephens, J. D. (2008). Partisan politics, the welfare state, and three worlds of human capital formation. Comparative Political Studies, 41(4/5), 600-637.

Jakobi, A.P. (2011). Political parties and the institutionalization of education: a comparative analysis of party manifestos. Comparative Education Review, 55(2), 189-209.

Jakobi, A.P., \& Teltemann, J. (2011). Convergence in education policy? A quantitative analysis of policy change and stability in OECD countries. Compare, 41(5), 579-595.

Jakobi, A.P., Martens, K., \& Wolf, K.D. (2010). A governance perspective on education policy. In A.P. Jakobi, K. Martens \& K.D. Wolf (Hrsg.), Education in political science. Discovering a neglected field (S. 1-20). Abingdon: Routledge.

Jakobi, A.P., Teltemann, J., \& Windzio, M. (2010a). The internationalization of education: policy in a cross-national perspective. In K. Martens, A. Nagel, M. Windzio \& A. Weymann (Hrsg.), Transformation of education policy. National responses to the PISA study and the bologna process (S. 227-258). London: Palgrave Macmillan.

Jensen, C. (2011). Capitalist systems, deindustrialization, and the politics of public education. Comparative Political Studies, 44(4), 412-435.

Jesson, J. K., Matheson, L., \& Lacey, F. M. (2011). Doing your literature review - traditional and systematic techniques. Los Angeles: SAGE. 
Kaufmann, K., Reichart, E., \& Schömann, K. (2014). Der Beitrag von Wohlfahrtsstaatsregimen und Varianten kapitalistischer Wirtschaftssysteme zur Erklärung von Weiterbildungsteilnahmestrukturen bei Ländervergleichen. Report - Zeitschrift Für Weiterbildungsforschung, 37(2), 39-54.

Knauber, C., \& Ioannidou, A. (2016). Politiken der Grundbildung im internationalen Vergleich. Von der Politikformulierung zur Implementierung. Zeitschrift für Weiterbildungsforschung - Report, 39(2), 131-148.

Knill, C. (2005). Introduction: cross-national policy convergence: concepts, approaches and explanatory factors. Journal of European Public Policy, 12(5), 764-774.

Markowitsch, J., Käpplinger, B., \& Hefler, G. (2013). Firm-provided training in Europe and the limits of national skills strategies. European Journal of Education, 48(2), 281-291.

Martens, K., Rusconi, A., \& Leuze, K. (Hrsg.). (2007). New arenas of education governance: the impact of international organizations and markets on educational policy making. Basingstoke Hampshire: Palgrave Macmillan.

Martin, A., \& Rüber, I.E. (2016). Die Weiterbildungsbeteiligung von Geringqualifizierten im internationalen Vergleich: Eine Mehrebenenanalyse. Zeitschrift für Weiterbildungsforschung - Report, 39(2), 149-169.

Nagel, A. (2010). Comparing education policy networks. In K. Martens, A. Nagel, M. Windzio \& A. Weymann (Hrsg.), Transformations of the state. Transformation of education policy (S. 199-226). Basingstoke: Palgrave Macmillan.

Nikolai, R. (2007). Die Bildungsausgaben der Schweiz im intranationalen und internationalen Vergleich. Berlin: Dissertation.de.

Nikolai, R., \& Ebner, C. (2013). Zur Verknüpfung von Berufsbildung und Hochschulbildung: Reformen in Deutschland, Österreich und der Schweiz. In K. Amos, J. Schmid, J. Schrader \& A. Thiel (Hrsg.), Kultur - Ökonomie - Globalisierung. Eine Erkundung von Rekalibrierungsprozessen in der Bildungspolitik (S. 63-80). Baden-Baden: Nomos.

North, D. C. (1990). Institutions, institutional change and economic performance. Cambridge: Cambridge University Press.

Pechar, H., \& Andres, L. (2011). Higher-education policies and welfare regimes: international comparative perspectives. Higher Education Policy, 24(1), 25-52.

Petticrew, M., \& Roberts, H. (2006). Systematic reviews in the social sciences - a practical guide. Malden Oxford Carlton: Blackwell.

Roosmaa, E.-L., \& Saar, E. (2012). Participation in non-formal learning in EU-15 and EU-8 countries: demand and supply side factors. International Journal of Lifelong Education, 31(4), 477-501.

Rubenson, K. (2006). The Nordic model of lifelong learning. Compare, 36(3), 327-341.

Rubenson, K., \& Desjardins, R. (2009). The impact of welfare state regimes on barriers to participation in adult education. Adult Education Quarterly, 59(3), 187-207.

Scharpf, F.W. (2006). Interaktionsformen. Akteurzentrierter Institutionalismus in der Politikforschung. Wiesbaden: VS.

Schemmann, M. (2007). Internationale Weiterbildungspolitik und Globalisierung. Orientierungen und Aktivitäten von OECD, EU, UNESCO und Weltbank. Bielefeld: wbv.

Schmid, J., Amos, K., Schrader, J., \& Thiel, A. (Hrsg.). (2011). Welten der Bildung? Vergleichende Analysen von Bildungspolitik und Bildungssystemen. Baden-Baden: Nomos.

Schmidt, M.G. (2007). Testing the retrenchment hypothesis: educational spending, 1960-2002. In F.G. Castles (Hrsg.), The disappearing state? Retrenchment realities in an age of globalisation (S. 83-159). Cheltenham: Edward Elgar.

Schrader, J. (2011). Struktur und Wandel der Weiterbildung. Bielefeld: wbv.

Souto-Otero, M., \& Ure, O. B. (2012). The coherence of vocational education and training in Norway and Spain: national traditions and the reshaping of VET governance in hybrid VET systems. Compare, 42(1), 91-111.

Trampusch, C. (2009). Europeanization and institutional change in vocational education and training in Austria and Germany. Governance, 22(3), 369-395.

Trampusch, C. (2010). Co-evolution of skills and welfare in coordinated market economies? A comparative historical analysis of Denmark, the Netherlands and Switzerland. European Journal of Industrial Relations, 16(3), 197-220.

Trampusch, C. (2010a). Employers, the state and the politics of institutional change: vocational education and training in Austria, Germany and Switzerland. European Journal of Political Research, 49(4), $545-573$.

Tsebelis, G. (1995). Decision making in political systems: veto players in presidentialism, parliamentarism, multicameralism and multipartism. British Journal of Political Science, 25(3), 289-325. 
Vogtenhuber, S. (2015). Explaining country variation in employee training: an institutional analysis of education systems and their influence on training and its returns. European Sociological Review, 31(1), 77-90.

Wenzelburger, G., \& Zohlnhöfer, R. (Hrsg.). (2015). Handbuch Policy-Forschung. Wiesbaden: Springer VS.

Wenzelburger, G. (2015). Parteien. In G. Wenzelburger \& R. Zohlnhöfer (Hrsg.), Handbuch Policy-Forschung (S. 81-112). Wiesbaden: Springer VS.

Wiborg, S. (2013). Neo-liberalism and universal state education: the cases of Denmark, Norway and Sweden 1980-2011. Comparative Education, 49(4), 407-423.

Wiborg, S. (2015). Privatizing education: free school policy in Sweden and England. Comparative Education Review, 59(3), 473-497.

Wilensky, H. (1975). The welfare state and equality. Structural and ideological roots of public expenditures. London: SAGE.

Wilkoszewski, H., \& Sundby, E. (2016). From hard to soft governance in multi-level education systems. European Journal of Education, 51(4), 447-462.

Willemse, N., \& de Beer, P. (2012). Three worlds of educational welfare states? A comparative study of higher education systems across welfare states. Journal of European Social Policy, 22(2), 105-117.

Witte, J., van der Wende, M., \& Huisman, J. (2008). Blurring boundaries: how the Bologna process changes the relationship between university and the non-university higher education in Germany, the Netherlands and France. Studies in Higher Education, 33(2), 217-231.

Wolf, F. (2009). The division of labour in education funding: a cross-national comparison of public and private education expenditure in 28 OECD countries. Acta Politica, 44(1), 50-73.

Wolf, F., \& Zohlnhöfer, R. (2009). Investing in human capital? The determinants of private education expenditure in 26 OECD countries. Journal of European Social Policy, 19(3), 230-244.

Zehavi, A. (2012). Welfare state politics in privatization of delivery. Comparative political studies, 45(2), 194-219. 